\title{
Classification of singular germs of mappings and deformations of compact surfaces of class $\mathrm{VII}_{0}$
}

\author{
by Georges Dloussky (Marseille) and Franz Kohler (Angers)
}

\begin{abstract}
We classify generic germs of contracting holomorphic mappings which factorize through blowing-ups, under the relation of conjugation by invertible germs of mappings. As for Hopf surfaces, this is the key to the study of compact complex surfaces with $b_{1}=1$ and $b_{2}>0$ which contain a global spherical shell. We study automorphisms and deformations and we show that these generic surfaces are endowed with a holomorphic foliation which is unique and stable under any deformation.
\end{abstract}

Surface means a complex manifold of dimension 2. We denote by $b_{i}(S)$ the $i$ th Betti number of $S$.

0. Introduction. Classification of compact complex surfaces $S$ without non-constant meromorphic functions with Betti numbers $b_{1}(S)=1$ and $b_{2}(S)=0$ is now well known thanks to K. Kodaira [KO], M. Inoue [I], Inoue-Kobayashi-Ochiai [IKO], F. Bogomolov [B], Li-Yau-Zheng [LYZ] and A. Teleman $[\mathrm{T}]$. Roughly speaking, there are two kinds of surfaces: those with at least one curve, Hopf surfaces, and those without curves, Inoue surfaces $S_{M}, S_{N ; p, q, r ; t}^{(+)}$and $S_{N ; p, q, r}^{(-)}$. All these surfaces admit global foliations.

The first step has been the study of Hopf surfaces, initiated by K. Kodaira $[\mathrm{KO}]$, based on the classification of invertible contracting mappings from $\left(\mathbb{C}^{2}, 0\right)$ to $\left(\mathbb{C}^{2}, 0\right)$ and their normal forms. It is a preliminary result and the proofs which are computational are relatively easy because the formal classification is the same as the analytic classification. Primary Hopf surfaces which are homeomorphic to the product $S^{1} \times S^{3}$ of spheres are particular cases of a wider class of non-kählerian compact complex surfaces, the class of surfaces containing a global spherical shell (GSS), i.e. surfaces in which there is an open embedding of a neighbourhood of $S^{3}$ in $\mathbb{C}^{2}$ which does not disconnect the surface.

1991 Mathematics Subject Classification: 32J15, 32G05, 32G13, 32Mxx.

Key words and phrases: surfaces of class $\mathrm{VII}_{0}$, germs of mappings. 
In the same way there is a wider class of contracting mappings, that is to say, mappings $F$ which can be factorized through a finite sequence of blowing-ups, i.e. $F=\Pi \sigma$, where $\Pi=\Pi_{0} \ldots \Pi_{n-1}$ is a sequence of $n \geq 0$ blowing-ups and $\sigma$ is a germ of isomorphism (when $n=0$ we recover Hopf surfaces); the corresponding surface $S=S(F)$ satisfies $b_{2}(S)=n$. Since all known surfaces with $b_{2}>0$ contain GSS, the strategy is to repete the one which has been successful for $b_{2}=0$. This study initiated in [D1] and [D2] has to overcome two difficulties: for computations it is not possible to write down a general form of the objects, because there are an enormous number of possible cases; the second one comes from the fact that the formal classification is weaker than the analytic classification. Therefore, the method is to work out simultaneously the classification of germs and the classification of surfaces, good parameters of the germs (local objects) corresponding to generators of the cohomology space $H^{1}(S, \Theta)$ and thus to versal deformations of the compact surfaces (global objects) $S$, where it is possible to apply the general tools of analytic geometry.

In the case $b_{2}(S)>0$, it is an open question whether there exists a curve, but by [N1], if there are curves, they are necessarily rational or non-singular elliptic, as in the GSS case; moreover (see [N2]), when the surface contains exactly $b_{2}(S)$ rational curves (I. Nakamura calls them special surfaces), then the intersection matrix $M(S)$ is equal to the intersection matrix $M\left(S^{\prime}\right)$ of a surface $S^{\prime}$ with GSS, and these surfaces are deformation equivalent. In the latter case conjecture 2 (below) would imply that $S$ contains a GSS. Complex non-singular foliations cannot exist when $b_{2}>0$ because of the Baum-Bott formula (see $[\mathrm{BR}]$ ). It is expected that singular foliations play an important role; in surfaces with a GSS, rational curves are recovered from invariant curves of the foliation at singular points (such invariant curves always exist by the Camacho-Sad theorem).

This article is devoted to surfaces with GSS which satisfy $\operatorname{tr}(S) \neq 0$, i.e. Inoue surfaces or generic surfaces. We refer to Section 1.1 for basic constructions and definitions.

The classification is carried out in $\S 1$ for generic germs and Inoue germs. With each germ $F$ there is associated an invariant formal curve which is divergent if and only if $F$ is not an Inoue germ; a step of the proof consists in the convergent classification of these divergent objects. As a consequence, we prove the existence of a unique foliation which is, on that complement of the rational curves, the affine bundle structure of [E1]; in particular the leaves in that complement are isomorphic to $\mathbb{C}$. A noticeable fact is that the attraction basins of generic germs are Fatou-Bieberbach domains (Remark 1.25).

In $\S 2$ explicit semi-universal deformations are given. It appears that the good parameters are the blown-up points and another one, the trace of the tangent mapping at the fixed point $D F(0)$. As a consequence, we recover 
the Enoki theorem [E2] for a surface $S$ with $\operatorname{tr}(S) \neq 0$. This section is somewhat technical, and yields the stability of the unique foliation on $S$ in its versal deformation. In $\S 3$, we describe the group of automorphisms which leave the irreducible curves globally invariant. We define a marked surface to be a surface with a rational curve chosen. The aim of this notion is to restrict the automorphism group. We obtain moduli of marked surfaces and a universal logarithmic deformation for generic marked surfaces. We postpone to another article the study of quotients of surfaces with non-trivial automorphism group.

It has to be pointed out that our methods are based on normal forms of germs of singular mappings $F$, therefore we have to suppose the existence of a GSS; this is less general than the approach of I. Enoki [E1], [E2], or I. Nakamura [N1], [N2], but is more precise. Moreover, it appeared recently that there are relations between $\mathrm{VII}_{0}$-class surfaces and dynamical systems in dimension two through contracting germs: for example the first author noticed that compact complex surfaces obtained in $[\mathrm{H}]$ by adding rational curves to the quotient surface $U_{+} / \mathbb{Z}$, where $U_{+}$is the open set of points $z$ of $\mathbb{C}^{2}$ such that the iterated images $H^{p}(z)$ of $z$ by an Hénon mapping $H(x, y)=(p(x)-a y, x)$ tend to $\infty$, contain GSS. In fact it is a surface with $\operatorname{tr}(S)=0$ endowed with a global foliation and in our notations $U_{+}=\widetilde{S} \backslash \widetilde{D}$ is the complement of the rational curves in the universal covering space of $S$.

We end by giving three conjectures in the general case.

Conjecture 1 . Any germ $F=\Pi \sigma$ is conjugate to a polynomial mapping.

Conjecture 2 . Let $\mathcal{S} \rightarrow B$ be a family of compact holomorphic surfaces with $B$ a connected manifold. If there is $u \in B$ such that $S_{u}$ contains a GSS, then all the surfaces contain a GSS.

ConjeCture 3 . If $S$ contains a GSS then $S$ admits a holomorphic singular foliation with leaves isomorphic to $\mathbb{C}$ or $\mathbb{C}^{\star}$.

\section{Normal forms of germs of mappings with non-vanishing trace}

1.1. Basic constructions. In this subsection, notations and results come from [D1], and we refer to that paper for proofs and details. Let $F=\Pi \sigma=$ $\Pi_{0} \ldots \Pi_{n-1} \sigma:\left(\mathbb{C}^{2}, 0\right) \rightarrow\left(\mathbb{C}^{2}, 0\right)$ be a germ of mapping where $\Pi_{i}$ is the blowing-up of the point $O_{i-1}$ and $O_{i} \in C_{i}=\Pi_{i}^{-1}\left(O_{i-1}\right)$ for $0 \leq i \leq n-1$, $O_{-1}=0 \in \mathbb{C}^{2}$, and $\sigma$ is a germ of isomorphism with $\sigma(0)=O_{n-1}$.

With such a germ one associates a compact complex surface in the following way: $\sigma$ is defined on a neighbourhood of 0 , say a neighbourhood of the closed unit ball $\bar{B} \subset \mathbb{C}^{2}$, so we have a sequence of blowing-ups over the 
ball $B$ :

$$
B_{n-1} \stackrel{\Pi_{n-1}}{\longrightarrow} \cdots \longrightarrow B_{i} \stackrel{\Pi_{i}}{\longrightarrow} B_{i-1} \longrightarrow \cdots \longrightarrow B_{0} \stackrel{\Pi_{0}}{\longrightarrow} B .
$$

It will always be supposed that $\sigma(B)$ is relatively compact in $B_{n-1}$. If $B^{\prime}$ is a ball slightly smaller than $B$, and if we remove the closed ball $\overline{\sigma\left(B^{\prime}\right)}$, then it is possible to identify isomorphic neighbourhoods of $\Sigma=\Pi^{-1}(\partial B)$ and $\sigma(\partial B)$ by $\sigma \Pi$ in order to obtain a minimal compact complex surface with a GSS, denoted by $S=S(\Pi, \sigma)$, with Betti numbers satisfying $b_{1}(S)=1$ and $b_{2}(S)=n$. In $S(\Pi, \sigma)$, the exceptional curve of the first kind has been changed into a rational curve with self-intersection $\leq-2$ or a singular rational curve because the inverse image by $\sigma$ of the removed part of $C_{n-1}$ contains 0 , therefore is blown up at least once again. If the points $O_{i}$ are moved outside the rational curves $C_{i}$, then $S$ is no more minimal. If $F$ and $F^{\prime}$ are conjugate, that is to say, $F^{\prime}=\Pi^{\prime} \sigma^{\prime}=\varphi^{-1} F \varphi$ where $\varphi$ is a germ of isomorphism, then $\varphi$ induces an isomorphism between $S(\Pi, \sigma)$ and $S\left(\Pi^{\prime}, \sigma^{\prime}\right)$. Conversely, given a surface $S$ containing a GSS, M. Kato [KA] proved that $S$ may be obtained in this way. In fact if $b_{2}(S)=n$, there are $n$ classes of germs and $n$ homotopy classes of GSS.

The trace of a germ $F=\Pi \sigma$ (resp. of a surface $S(\Pi, \sigma)$ ) is by definition the trace $\operatorname{tr}(D F(O))$ of the tangent mapping $D F$ at the fixed point of $F$. The trace is independent of the choice of GSS and depends only on the isomorphism class of $S$, so it is denoted by $\operatorname{tr}(S)$. We have $0 \leq|\operatorname{tr}(S)|<1$ and $\operatorname{tr}(S) \neq 0$ if and only if one of the following equivalent conditions is satisfied:

(i) for every $0 \leq i \leq n-1, O_{i}$ is not in the intersection of $C_{i}$ with a strict transform of $C_{k}, k<i$, or of $\sigma^{-1}\left(C_{n-1}\right)$;

(ii) $S$ contains a cycle $\Gamma$ of rational curves such that $\Gamma^{2}=0$.

Let $F=\Pi \sigma$ be a germ such that $\operatorname{tr}(S) \neq 0$ and $S=S(F)$ be the associated minimal surface with $b_{2}(S)=n \geq 1$. The germ $F=\Pi \sigma$ (resp. the minimal surface $S$ ) is called an Inoue germ (resp. an Inoue surface) if one of the following equivalent conditions holds (see [D1] for the proof):

(a) there exists a germ of a curve $(\gamma, 0)$ such that $F_{\mid \gamma}:(\gamma, 0) \rightarrow(\gamma, 0)$ is an isomorphism;

(b) $F$ is conjugate to $N(z)=\left(t^{n} z_{1} z_{2}^{n}, t z_{2}\right)$;

(c) $S$ admits a global vector field;

(d) $S$ contains an elliptic curve.

A germ of mapping will be called generic if its trace is not vanishing and it is not an Inoue germ (this definition is slightly different from that of [KH1], [KH2] where generic means "with non-vanishing trace"). 
The universal covering space $(\widetilde{S}, \widetilde{\omega})$ of $S$ is obtained by glueing a sequence $\left(A_{i}\right)_{i \in \mathbb{Z}}$ of copies of the blown-up ball with a hole, the pseudoconcave boundary of $A_{i}$ being identified with the pseudoconvex boundary of $A_{i+1}$. The automorphism $\widetilde{g}: \widetilde{S} \rightarrow \widetilde{S}$ sends $A_{i}$ onto $A_{i+1}$. Now, in $\widetilde{S}$ there is countable family of rational curves with a canonical order induced by the blowing-ups, "the order of creation". If $\operatorname{tr}(S)=0$ this order is not obviously understandable from the graph of curves. Sometimes we shall denote by $C+1$ the curve obtained after $C$.

Now if we choose any curve $C$ in $\widetilde{S}$ we construct a new surface $\widehat{S}_{C}$ with a canonical morphism $p_{C}: \widetilde{S} \rightarrow \widehat{S}_{C}$ in the following way: If $C \subset \bigcup_{i \leq p} A_{i}$ we fill in the hole of $A_{p}$ with a ball, thus obtaining a surface with an exceptional curve of the first kind. If it is $C$ we have obtained $\widehat{S}_{C}$; if not, we blow down this exceptional curve, obtaining a new exceptional curve of the first kind, and so on till $C$ becomes such a curve. Finally, $\widehat{S}_{C}$ is obtained by blowing down a "half-infinite" number of curves onto a point $\widehat{O}_{C} \in C$.

The following construction relates compact surfaces with contracting germs: We notice that $\widetilde{g}$ induces an isomorphism $\sigma_{C}^{C+n}: \widehat{S}_{C} \rightarrow \widehat{S}_{C+n}$ and that there is a canonical mapping $\Pi_{C+n}^{C}: \widehat{S}_{C+n} \rightarrow \widehat{S}_{C}$ which blows down $n$ curves, such that $p_{C}=\Pi_{C+n}^{C} p_{C+n}$. Then $F_{C}:=\Pi_{C+n}^{C} \sigma_{C}^{C+n}$ is a contracting mapping with fixed point $\widehat{O}_{C}$. The following diagram is commutative:

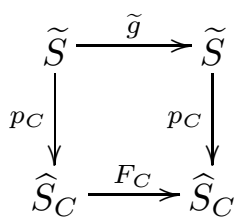

1.2. Normal germs. A generic germ or an Inoue germ can always be written in the form

$$
F(z)=\Pi \circ \sigma(z)=\left(\sigma_{1}(z) \sigma_{2}(z)^{n}+\sum_{i=0}^{n-1} \alpha_{i} \sigma_{2}(z)^{i+1}, \sigma_{2}(z)\right),
$$

where

$$
\Pi=\Pi_{0} \ldots \Pi_{n-1}, \quad \Pi_{i}\left(u_{i}, v_{i}\right)=\left(u_{i} v_{i}+\alpha_{i-1}, v_{i}\right)
$$

and

$$
\sigma(z)=\left(\sigma_{1}(z)+\alpha_{n-1}, \sigma_{2}(z)\right) .
$$

The point $O_{i}=\left(\alpha_{i}, 0\right)$ cannot be the infinite point of $C_{i}$ because of the nonzero trace hypothesis. It will be proved that by a well chosen conjugation the germ of isomorphism $\sigma$ satisfies $\sigma\left(z_{1}, z_{2}\right)=\left(\sigma_{1}(z), \sigma_{2}(z)\right)=\left(z_{1}, t z_{2}\right)$ where $t$ is the trace, $0<|t|<1$. But, to start with, it will be shown that in a given equivalence class this latter form is not unique. 
Definition 1.1. A germ of the form

$$
F(z)=\left(z_{1} z_{2}^{n} t^{n}+\sum_{i=0}^{n-1} \alpha_{i} t^{i+1} z_{2}^{i+1}, t z_{2}\right)
$$

is called a normal germ with non-vanishing trace. It will be called generic if not all $\alpha_{i}$ vanish.

Such germs are global automorphisms of $\mathbb{C} \times \mathbb{C}^{\star}$ and map affinely horizontal lines to horizontal lines; therefore the associated surface $S(F)$ has a foliation.

REMARK. The family of surfaces $S_{t, \alpha}^{n}=S(F)$ coincides with the family of surfaces $S_{n, t^{\prime}, \alpha^{\prime}}$ of [E1], where $n$ is the number of rational curves for both notations, $t=\alpha^{\prime}$ and $\left(\alpha_{0}, \ldots, \alpha_{n-2}, \alpha_{n-1}\right)=\left(t_{1}^{\prime}, \ldots, t_{n-1}^{\prime}, t_{0}^{\prime}\right)$.

LEMMA 1.2. If $F=\Pi \sigma$ is a normal generic germ with trace $t \neq 0$, then there exists an isomorphism

$$
f: \widehat{S}_{C} \backslash \bigcup_{C^{\prime} \neq C} C^{\prime} \rightarrow \mathbb{C}^{2}
$$

such that the following diagram is commutative:

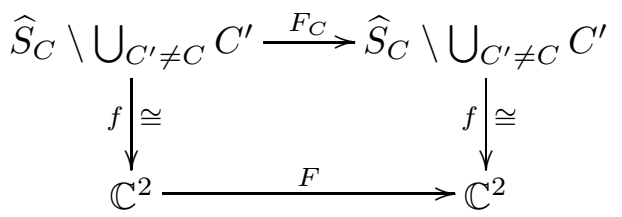

Proof. By construction of $\widehat{S}_{C}$ from $F$, there exists an isomorphism $f: U_{C} \rightarrow U$ from a neighbourhood of $\widehat{O}_{C}$ onto a neighbourhood of 0 such that the following diagram is commutative:

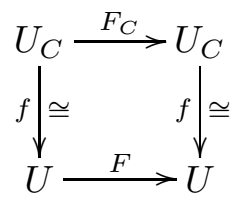

We have to extend $f$ : Let $z$ be in the complement of all the compact curves of $\widehat{S}_{C}$; there exists an integer $p \geq 0$ for which $F_{C}^{p}(z) \in U_{C}$; since $f(z)$ does not belong to the singular set of $F$ and $F$ is an automorphism of $\mathbb{C} \times \mathbb{C}^{\star}$, we define $f(z):=F^{-p} \circ f \circ F_{C}^{p}(z)$. Since $f=F^{-1} \circ f \circ F_{C}$, the image of $z$ does not depend on the choice of $p$. By the Riemann extension theorem we obtain the desired extension

$$
f: \widehat{S}_{C} \backslash \bigcup_{C^{\prime} \neq C} C^{\prime} \rightarrow \mathbb{C}^{2} .
$$


Since $f$ is an isomorphism between $\widehat{S}_{C} \backslash \bigcup C^{\prime}$ and $\mathbb{C} \times \mathbb{C}^{\star}$, $\operatorname{det} D f(z)$ could vanish only on $C$; but in a neighbourhood of $\widehat{O}_{C}, f$ is an isomorphism, thus $\operatorname{det} D f(z)$ never vanishes. Moreover, the image of $f$ contains $\mathbb{C} \times \mathbb{C}^{\star}$ because the attraction basin of 0 , i.e. the points $z$ such that the sequence $\left(F^{n}(z)\right)$ tends to 0 , is $\mathbb{C} \times \mathbb{C}^{\star}$. We conclude that $f$ is an isomorphism.

LEMMA 1.3. If $F, F^{\prime}:\left(\mathbb{C}^{2}, 0\right) \rightarrow\left(\mathbb{C}^{2}, 0\right)$ are two normal germs with nonvanishing trace, then any isomorphism germ $\varphi:\left(\mathbb{C}^{2}, 0\right) \rightarrow\left(\mathbb{C}^{2}, 0\right)$ which satisfies $\varphi \circ F=F^{\prime} \circ \varphi$ is convergent in $\mathbb{C}^{2}$.

Proof. The germ allows us to recover a surface $S$, its universal covering space $\widetilde{S}$ and the space obtained by the blowing-down of all curves greater than $C$, denoted by $\widehat{S}_{C}$. The lemma is an immediate consequence of Lemma 1.2 and the following commutative diagram:

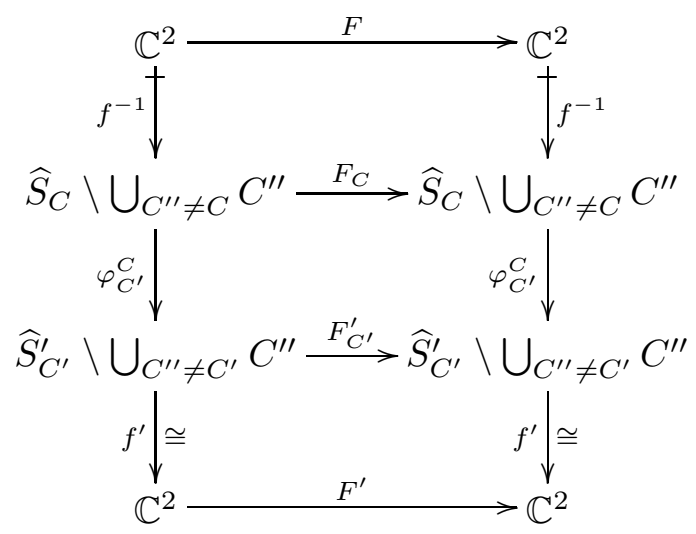

since $\varphi=f^{\prime} \circ \varphi_{C^{\prime}}^{C} \circ f^{-1}$.

THEOREM 1.4. Let

$$
\begin{gathered}
F(z)=\left(z_{1} z_{2}^{n} t^{n}+\sum_{i=0}^{n-1} \alpha_{i} t^{i+1} z_{2}^{i+1}, t z_{2}\right) \quad \text { and } \\
F^{\prime}(z)=\left(z_{1} z_{2}^{n} t^{\prime n}+\sum_{i=0}^{n-1} \alpha_{i}^{\prime} t^{i+1} z_{2}^{i+1}, t^{\prime} z_{2}\right)
\end{gathered}
$$

be normal germs.

(1) The germs $F$ and $F^{\prime}$ are conjugate, i.e. there exists $\varphi$ such that $\varphi F=F^{\prime} \varphi$, if and only if $t=t^{\prime}$ and there exists $\kappa \in \mathbb{C}^{\star}$ and $n$th root of unity $\lambda$ such that

$$
\left(\alpha_{0}^{\prime}, \ldots, \alpha_{i}^{\prime}, \ldots, \alpha_{n-1}^{\prime}\right)=\kappa\left(\alpha_{0}, \lambda \alpha_{1}, \ldots, \lambda^{i} \alpha_{i}, \ldots, \lambda^{n-1} \alpha_{n-1}\right) .
$$


In this case $\varphi$ is necessarily linear and $\varphi\left(z_{1}, z_{2}\right)=\left(a z_{1}, \lambda^{-1} z_{2}\right)$ with $a=k \lambda^{-1} \neq 0$ and $\lambda^{n}=1$. In particular, if $F(z)=N(z)=\left(z_{1} z_{2}^{n} t^{n}, t z_{2}\right)$, then $F^{\prime}=F$; if $n=1$, then there are only two classes.

(2) A germ of isomorphism $\varphi$ commutes with $F$ if and only if it satisfies the following conditions:

(a) If $\left(\alpha_{0}, \ldots, \alpha_{i}, \ldots, \alpha_{n-1}\right)=(0, \ldots, 0)$, i.e. $F=N$, then $\varphi\left(z_{1}, z_{2}\right)=$ $\left(a z_{1}, \lambda^{-1} z_{2}\right)$ where $a \neq 0$ and $\lambda^{n}=1$.

(b) If there exists exactly one index $j$ such that $\alpha_{j} \neq 0$, then $\varphi\left(z_{1}, z_{2}\right)=$ $\left(\lambda^{-(j+1)} z_{1}, \lambda^{-1} z_{2}\right)$ where $\lambda^{n}=1$.

(c) If there are at least two indices $j$ and $k$ such that $\alpha_{j} \neq 0$ and $\alpha_{k} \neq 0$, let $1 \leq m \leq n-1$ be the least integer such that $m=k-j \bmod n$. Then $\varphi(z)=\left(\lambda^{-m} z_{1}, \lambda^{-1} z_{2}\right)$ with $\lambda^{\operatorname{gcd}\{m, n\}}=1$. In particular, generically $\varphi=$ Id.

Proof. (1) Let $\varphi(z)=\left(\sum a_{i j} z_{1}^{i} z_{2}^{j}, \sum b_{i j} z_{1}^{i} z_{2}^{j}\right)$ be a germ of isomorphism which satisfies the equation $\varphi F=F^{\prime} \varphi$. It yields

$$
\sum b_{i j}\left(z_{1} z_{2}^{n} t^{n}+\sum_{k=0}^{n-1} \alpha_{k} t^{k+1} z_{2}^{k+1}\right)^{i} t^{j} z_{2}^{j}=t^{\prime}\left(\sum b_{i j} z_{1}^{i} z_{2}^{j}\right) .
$$

The identification of the linear parts of (1.5) and induction on the degree of the homogeneous parts of $\varphi_{2}$ show that $t=t^{\prime}$ and $\varphi_{2}(z)=\lambda z_{2}$.

Now, we consider the equation analogous to (1.5) given by the first members of the conjugation relation:

$$
\begin{aligned}
\sum a_{i j}\left(z_{1} z_{2}^{n} t^{n}+\sum_{k=0}^{n-1} \alpha_{k} t^{k+1} z_{2}^{k+1}\right)^{i} t^{j} z_{2}^{j} & \\
& =\left(\sum a_{i j} z_{1}^{i} z_{2}^{j}\right) \lambda^{n} z_{2}^{n} t^{n}+\sum_{i=0}^{n-1} \alpha_{i}^{\prime} t^{i+1} \lambda^{i+1} z_{2}^{i+1}
\end{aligned}
$$

Then:

a) The comparison of coefficients of terms which contain $z_{1} z_{2}^{n}$ yields $\lambda^{n}=1$.

b) The comparison of the terms of total degree $n+p \bmod z_{2}^{n+p}$, for $p \geq 2$, allows one to prove by induction on $p$ that

$$
a_{i j}=0 \quad \text { for } i+j=p \geq 2, i \geq 1 \text {. }
$$

Therefore

$$
\varphi_{1}\left(z_{1}, z_{2}\right)=a_{10} z_{1}+\sum_{j=1}^{\infty} a_{0 j} z_{2}^{j}
$$


By substitution we have from (1.6)

$$
\begin{aligned}
& a_{10}\left(z_{1} z_{2}^{n} t^{n}+\sum_{k=0}^{n-1} \alpha_{k} t^{k+1} z_{2}^{k+1}\right)+\sum_{j=1}^{\infty} a_{0 j} t^{j} z_{2}^{j} \\
& \quad=\left(a_{10} z_{1}+\sum_{j=1}^{\infty} a_{0 j} t^{j} z_{2}^{j}\right) z_{2}^{n} t^{n}+\sum_{k=0}^{n-1} \alpha_{k}^{\prime} t^{k+1} \lambda^{k+1} z_{2}^{k+1}
\end{aligned}
$$

and by cancellation

$a_{10}\left(\sum_{k=0}^{n-1} \alpha_{k} t^{k+1} z_{2}^{k+1}\right)+\sum_{j=1}^{\infty} a_{0 j} t^{j} z_{2}^{j}=\sum_{j=1}^{\infty} a_{0 j} t^{j+n} z_{2}^{j+n}+\sum_{k=0}^{n-1} \alpha_{k}^{\prime} t^{k+1} \lambda^{k+1} z_{2}^{k+1}$.

An easy computation shows that

$$
a_{0 i}= \begin{cases}-a_{10} \alpha_{i-1}+\alpha_{i-1}^{\prime} \lambda^{i} & \text { for } i=1, \ldots, n, \\ a_{0, i-n} & \text { for } i>n .\end{cases}
$$

We deduce from (1.7) that if one of the $a_{0 i}, 1 \leq i \leq n$, is not zero then the radius of convergence of the series $\sum_{j>0} a_{0 j} z_{2}^{j}$ is 1 . But by Lemma 1.3, $\varphi$ should converge on $\mathbb{C}^{2}$, therefore $a_{0 j}=0$ for all $j \geq 1$. Since $a_{10} \neq 0$, we obtain the first assertion (1) with $\kappa=a_{10} \lambda^{-1}$ and $\lambda$ replaced by $\lambda^{-1}$. So $\varphi(z)=\left(a z_{1}, \lambda^{-1} z_{2}\right)$ with $a=k \lambda^{-1} \neq 0$ and $\lambda^{n}=1$.

(2) is a straightforward consequence of the first assertion.

We are now going to show that every germ with non-vanishing trace is equivalent to a normal germ.

Proposition 1.8. Let $S$ be a surface with $\operatorname{tr}(S) \neq 0$. Denote by $\widetilde{C}$ the union of all rational curves of the universal covering space $\widetilde{S}$ of $S$. Then:

(i) there exist a holomorphic function $\widetilde{w}$ on $\widetilde{S}$ and $t \in \mathbb{C}^{\star}$ with $|t|<1$ such that $\operatorname{divisor}(\widetilde{w})=\widetilde{C}$ and $\widetilde{w} \circ \widetilde{g}=t \widetilde{w}$;

(ii) $t=\operatorname{tr}(S)$;

(iii) for any rational curve $C$ in $\widetilde{S}$, the germ $F_{C}$ is equivalent to a germ $F=\left(F_{1}, F_{2}\right)$ with $F_{2}(z)=t z_{2}$.

Pro of. The universal covering space $\widetilde{S}$ is the union of annuli and a generator of the fundamental group is given by a path joining the two connected components of the boundary of an annulus. Therefore the same arguments as in Lemma 4.7 of [E2] give (i); however, to make the paper self-contained we give a simplified proof:

By Theorem 3 of $[\mathrm{KO}]$, part I, $b_{1}(S)=h^{10}+h^{01}$, thus we have the exact sequence

$$
0 \rightarrow H^{0}(S, d \mathcal{O}) \rightarrow H^{1}(S, \mathbb{C}) \rightarrow H^{1}(S, \mathcal{O}) \rightarrow 0 .
$$


The maximal divisor $D$ of $S$ satisfies $c_{1}([D])^{2}=D^{2}=0$. Since $b_{-}=b_{2}(S)$, the complex Chern class $c_{1}([D])_{\mathbb{C}}$ vanishes. Using the commutative diagram

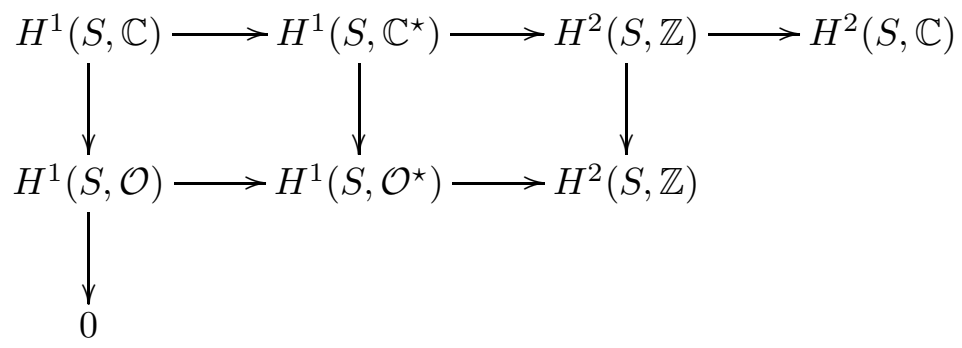

we conclude that $[D]$ is in the image of $H^{1}\left(S, \mathbb{C}^{\star}\right)$ in $H^{1}\left(S, \mathcal{O}^{\star}\right)$. This means that we may choose a covering $\mathcal{U}=\left(U_{i}\right)$ of $S$, defining functions $f_{i} \in \mathcal{O}\left(U_{i}\right)$ of $D$ and complex numbers $\lambda_{i j} \in \mathbb{C}^{\star}$ such that $f_{i}=\lambda_{i j} f_{j}$ for all $i, j$. Let $F=\left(\lambda_{i j}\right)$ be the corresponding flat line bundle. Since $F$ induces on $\widetilde{S}$ a holomorphically trivial bundle $\widetilde{F}=\widetilde{\omega}^{\star} F$, the section $\left(f_{i}\right)$ of $F$ induces a global holomorphic function $w$ on $\widetilde{S}$ which satisfies the desired conditions with $t \in \mathbb{C}^{\star}$. If $|t|=1$, then $w$ induces on $S$ a psh function and this is impossible. By the maximum principle $|t|<1$.

The assertion (ii) is an immediate consequence of (iii).

(iii) The function $\widetilde{w}$ on $\widetilde{S}$ induces a holomorphic function on $\widehat{S}_{C}$ denoted by $w$ where $C=\left\{z_{2}=0\right\}$ in a neighbourhood of $\widehat{O}_{C}$. By the commutativity of the diagram

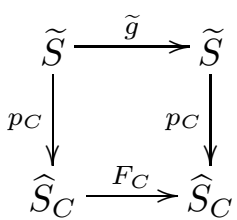

we have $w \circ F_{C}=t w$. We define $\varphi(z)=\left(z_{1}, w(z)\right)$. Since $w$ vanishes of order one on $C$, and does not vanish in the complement of $C, \varphi$ is an isomorphism and satisfies

$$
\varphi F_{C} \varphi^{-1}(z)=\left(\star, w \circ F_{C}\left(\varphi^{-1}(z)\right)\right)=\left(\star, t w\left(\varphi^{-1}(z)\right)\right)=\left(\star, t z_{2}\right) .
$$

THEOREM 1.9. Let $S$ be a minimal compact surface containing a GSS with $b_{2}(S)>0$. If $\operatorname{tr}(S) \neq 0$, then:

(i) $S$ admits a unique singular holomorphic foliation such that the singular points of the foliation are exactly the intersection points of the rational curves;

(ii) the complement of the singular points in a rational curve is a leaf with hyperbolic holonomy;

(iii) all the other leaves are isomorphic to $\mathbb{C}$ and accumulate to the cycle of rational curves of $S$; in particular, the closure of a leaf always contains a singular point; 
(vi) the foliation is defined by a global meromorphic 1-form with a logarithmic pole.

Proof. (i) By Proposition $1.8, F$ maps $\left\{z_{2}=\lambda\right\}$ onto $\left\{z_{2}=t \lambda\right\}$, therefore $S$ is endowed with a foliation which is unique by Theorem 2.4.1 of [KH3] and the fact that in any neighbourhood of an Inoue surface in its versal deformation there are all generic surfaces.

(ii) is evident.

(iii) Choose a leaf $\mathcal{L}$ in $S$. It induces a leaf $\widetilde{\mathcal{L}}$ in the universal covering space $\widetilde{S}=\bigcup A_{i}$. The image of $\widetilde{\mathcal{L}} \cap A_{i}$ in the ball is contained in the piece of line $L_{i}=\left\{z_{2}=\lambda\right\}$. There are two possibilities:

- $L_{i}$ does not meet $F(B)$, i.e. it is a disc and $\widetilde{\mathcal{L}} \cap A_{j}$ is empty for $j>i$ since $\left\{z_{2}=\lambda\right\}$ in $A_{i}$ is glued to $\left\{z_{2}=t^{-1} \lambda\right\}$ in $A_{i+1}$. For the same reason $L_{j}$ for $j<i$ are all dimension one annuli $r_{j}<\left|z_{1}\right|<1$ where the sequence $\left(r_{j}\right)$ is bounded from above, therefore $\mathcal{L}$ and $\widetilde{\mathcal{L}}$ are Riemann surfaces isomorphic to $\mathbb{C}$.

- $L_{i}$ meets $F(B)$, but there exists $j>i$ such that $L_{j}$ does not meet $F(B)$, and therefore we obtain the same conclusion.

(iv) $\omega=d z_{2} / z_{2}$ has the expected property.

In the following lemma we still denote by $C$ the image of $C \subset \widetilde{S}$ under the canonical mapping $p_{C}: \widetilde{S} \rightarrow \widehat{S}_{C}$. The function $\widehat{w}$ is induced by $\widetilde{w}$ of Proposition 1.8.

Lemma 1.10. Given a curve $C$ in $\widetilde{S}$, set $\{\infty\}=C \cap \bigcup_{C^{\prime}<C} C^{\prime}$. Then there exists $\varepsilon>0$ and an isomorphism

$$
f: U_{C}(\varepsilon):=\{|\widehat{w}(z)|<\varepsilon\} \backslash \bigcup_{C^{\prime}<C} C^{\prime} \rightarrow \mathbb{C} \times \Delta(\varepsilon)
$$

from a neighbourhood of $C \backslash\{\infty\}$ in $\widehat{S}_{C}$ onto $\mathbb{C} \times \Delta(\varepsilon)$, which maps $\{\widehat{w}=\delta\}$ to $\mathbb{C} \times\{\delta\}$.

Proof. We choose a spherical shell of $S$ such that $S$ is obtained from a blown-up neighbourhood of $\widehat{O}_{C}$ isomorphic to the unit ball $B$, in which $C=\left\{z_{2}=0\right\}$, and $\widehat{S}_{C}=\left(\bigcup_{i<0} A_{i}\right) \cup B$, where $A_{i}$ are copies of the blownup ball $B$ with image $\sigma(B)$ removed. If $U_{0}=B, U_{p}:=\bigcup_{-p<i<0} A_{i} \cup B$, $U_{p}^{\prime}=U_{p} \backslash \bigcup_{C^{\prime} \leq C} C^{\prime}$ then $\left(U_{p}\right)_{p \geq 0}$ and $\left(U_{p}^{\prime}\right)_{p \geq 0}$ are increasing sequences of open subsets of $\widehat{S}_{C}$. Thanks to Proposition 1.8(iii), we may suppose that $\widehat{w}(z)=z_{2}$ and

$$
F_{C \mid B}(z)=F(z)=\Pi \sigma(z)=\left(\sigma_{1}(z) z_{2}^{n} t^{n}+\sum_{i=0}^{n-1} \alpha_{i} t^{i+1} z_{2}^{i+1}, t z_{2}\right) .
$$


Choose $\varepsilon$ such that for $\lambda \neq 0$ with $|\lambda|<\varepsilon,\left\{z_{2}=\lambda\right\} \cap B \backslash F(B)$ is a topological annulus of dimension 1. The annulus $\left\{z_{2}=\lambda\right\}$ in $A_{i}$ is glued to $\left\{z_{2}=t^{-1} \lambda\right\}$ in $A_{i+1}$ which is an annulus or a disc. For every $p \geq 0, U_{p}^{\prime}$ is isomorphic to $B \backslash\{w=0\}$; here $U_{0}^{\prime}=B \backslash\{w=0\}$ is identified with $F^{p}(B) \backslash\{0\}$. Replacing, if necessary, $F$ by a conjugate of $F$ by a linear map $\varphi(z)=\left(z_{1}+A z_{2}, z_{2}\right)$ we may suppose that $\alpha_{1} \neq 0$, therefore in a neighbourhood of $z=(0,0)$,

$$
\sigma_{1}\left(z_{1}, z_{2}\right) z_{2}^{n} t^{n}+\sum_{i=0}^{n-1} \alpha_{i} t^{i+1} z_{2}^{i+1} \sim \alpha_{0} t z_{2} .
$$

Therefore if the $p$ th iteration of $F$ is $F^{p}=\left(F_{1}^{p}, F_{2}^{p}\right)$, then

$$
F_{1}^{p}\left(z_{1}, z_{2}\right) \sim \alpha_{0} t^{p} z_{2}
$$

We define

$$
f_{p}=\left(g_{p}, w\right): U_{p}^{\prime} \cap\{|w|<1\} \rightarrow \mathbb{C} \times \Delta^{\star}, \quad\left(z_{1}, z_{2}\right) \mapsto\left(\frac{z_{1}}{\alpha_{0} t^{p}}, w(z)\right) .
$$

The sequence $\left(f_{p}\right)$ is uniformly bounded on $U_{m}^{\prime}$ identified with $F^{p-m}(B) \backslash\{0\}$ in $U_{p}$. In fact, for $z \in B, A>1$ and $p \rightarrow \infty$ we have

$$
\left|g_{p}(z)\right|=\frac{\left|F_{1}^{p-m}(z)\right|}{\left|\alpha_{0}\right| \cdot|t|^{p}} \leq A|t|^{-m} .
$$

By Montel's theorem and a diagonal argument, there exists a convergent subsequence. Let $f$ be the limit. On $U_{m}^{\prime}, f=\left(g\left(z_{1}\right), w\left(z_{2}\right)\right)$ is bounded, thus by Riemann's extension theorem, $f$ extends across $C$. The leaves of the foliation are sent by $f$ to lines $\left\{z_{2}=\lambda\right\}$ in $\mathbb{C} \times \Delta$. Because of (1.11), $g$ is a non-zero linear map. Therefore $f$ is an isomorphism.

We are now in a position to find a simpler element in the conjugacy class of $F$ :

Lemma 1.12. Let

$$
F_{C}(z)=F(z)=\Pi \sigma(z)=\left(\sigma_{1}(z) z_{2}^{n} t^{n}+\sum_{i=0}^{n-1} \alpha_{i} t^{i+1} z_{2}^{i+1}, t z_{2}\right)
$$

be a germ of mapping with non-zero trace. Then there exists a germ of isomorphism $f$ such that

$$
F^{\prime}(z)=f F f^{-1}(z)=\left(\left(z_{1} a\left(z_{2}\right)+b\left(z_{2}\right)\right) z_{2}^{n} t^{n}+\sum_{i=0}^{n-1} \alpha_{i}^{\prime} t^{i+1} z_{2}^{i+1}, t z_{2}\right) .
$$

with $a \in \mathcal{O}^{\star}(\Delta)$. 
Pr o of. By Lemma 1.10, we have the following commutative diagram:

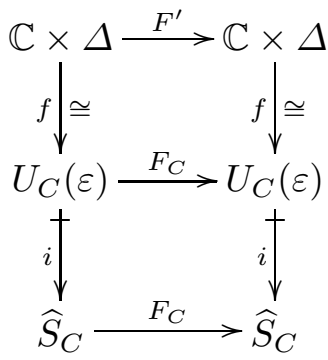

in which

$$
F^{\prime}(z)=\Pi^{\prime} \sigma^{\prime}(z)=\left(\sigma_{1}^{\prime}(z) z_{2}^{n} t^{n}+\sum_{i=0}^{n-1} \alpha_{i}^{\prime} t^{i+1} z_{2}^{i+1}, t z_{2}\right)
$$

and $\sigma_{1}^{\prime}$ is convergent in $\mathbb{C} \times \Delta$. Since $F_{1}^{\prime}\left(\cdot, z_{2}\right)$ is an automorphism of $\mathbb{C}$, $\sigma_{1}^{\prime}(z)=z_{1} a\left(z_{2}\right)+b\left(z_{2}\right)$ with a non-vanishing coefficient $a$.

This lemma establishes that in fact the foliation is the extension of a structure of affine bundle on the complement of the rational curves. Since $\operatorname{tr}(S) \neq 0$ if and only if there is a divisor $D$ on $S$ such that $D^{2}=0$ we obtain the main theorem of [E2] for surfaces containing a GSS:

Proposition 1.14. Let $S$ be a surface with $t=\operatorname{tr}(S) \neq 0$ and $D$ be the union of the rational curves of $S$. Let $E:=\mathbb{C}^{\star} /\left\{t^{p} \mid p \in \mathbb{Z}\right\}$ be the elliptic curve induced by the contraction $z_{2} \mapsto t z_{2}$ and $\omega: S \backslash D \rightarrow E$ the map induced by $w$. Then $(S \backslash D, \omega, E)$ is an affine bundle which is a linear bundle if and only if $S$ is an Inoue surface.

Lemma 1.15. Any germ $F$ with trace $t \neq 0$ is conjugate to a germ of the form

$$
F^{\prime}(z)=\left(\left(z_{1}+b^{\prime}\left(z_{2}\right)\right) z_{2}^{n} t^{n}+\sum_{i=0}^{n-1} \alpha_{i}^{\prime} t^{i+1} z_{2}^{i+1}, t z_{2}\right)
$$

with $b^{\prime}(0)=0$.

Pr o of. By Lemma 1.12, we may suppose that

$$
F(z)=\left(\left(z_{1} a\left(z_{2}\right)+b\left(z_{2}\right)\right) z_{2}^{n} t^{n}+\sum_{i=0}^{n-1} \alpha_{i} t^{i+1} z_{2}^{i+1}, t z_{2}\right) .
$$

By changing $\alpha_{n-1}$ if necessary, we may suppose that $b(0)=0$. Moreover, conjugation by an isomorphism $\varphi\left(z_{1}, z_{2}\right)=\left(z_{1}, \beta z_{2}\right)$ yields $a(0)=1$. We look for a germ of an isomorphism $\varphi\left(z_{1}, z_{2}\right)=\left(c\left(z_{2}\right) z_{1}, \beta z_{2}\right)$ which leaves invariant the fibres of the affine bundle. We have $c(0) \neq 0$. The equation 
$F^{\prime} \varphi=\varphi F$ gives, by (1.13),

$$
\begin{aligned}
& c\left(t z_{2}\right) a\left(z_{2}\right)=c\left(z_{2}\right) \beta^{n}, \\
& c\left(t z_{2}\right)\left(b\left(z_{2}\right) t^{n} z_{2}^{n}+\sum_{i=0}^{n-1} \alpha_{i} t^{i+1} z_{2}^{i+1}\right) \\
& \quad=b^{\prime}\left(\beta z_{2}\right) t^{n} z_{2}^{n} \beta^{n}+\sum_{i=0}^{n-1} \alpha_{i}^{\prime} t^{i+1} \beta^{i+1} z_{2}^{i+1} .
\end{aligned}
$$

Write $a\left(z_{2}\right)=1+a^{\prime}\left(z_{2}\right)$. Then (1.16) is solved by $\beta^{n}=1$ and the convergent infinite product

$$
c\left(z_{2}\right)=\prod_{i=0}^{\infty}\left(1+a^{\prime}\left(t^{i} z_{2}\right)\right) .
$$

The condition (1.17) is then easy to satisfy.

We want to show that the $\alpha_{i}$ are the good parameters and that we can choose the blown-up points, determined by the coefficients $\alpha_{i}, 0 \leq i \leq n-1$, in such a way that $b^{\prime}$ can be cancelled by a conjugation. For that purpose we describe the sequence $\left(O_{i}\right), i \geq 0$, of infinitely near points or equivalently the invariant formal curve of $F$ which exists by [D1], II.1.14. It is easy to check that formal curves are all formally isomorphic, but the isomorphism is in general not convergent. Moreover, if $F$ and $F^{\prime}$ are conjugate, then necessarily the formal curves are isomorphic. The idea of the following lemma is to classify these formal objects in a convergent way.

Lemma 1.18. Let $F$ be the germ

$$
F(z)=\left(\left(z_{1}+b\left(z_{2}\right)\right) z_{2}^{n} t^{n}+\sum_{i=0}^{n-1} \alpha_{i} t^{i+1} z_{2}^{i+1}, t z_{2}\right)
$$

with $b(0)=0$. Then there exist $\alpha_{i}^{\prime}, 0 \leq i \leq n-1$, such that $F$ is conjugate to

$$
F^{\prime}(z)=\left(z_{1} z_{2}^{n} t^{n}+\sum_{i=0}^{n-1} \alpha_{i}^{\prime} t^{i+1} z_{2}^{i+1}, t z_{2}\right)
$$

Proof. Let $\Gamma$ be the formal curve defined by the ideal $\left(z_{1}-\sum_{i=1}^{\infty} A_{i} z_{2}^{i}\right)$. Put $b\left(z_{2}\right)=\sum_{i=1}^{\infty} b_{i} z_{2}^{i}$. Then $\Gamma$ is an invariant curve if and only if

$$
\left(\sum_{i=1}^{\infty} A_{i} z_{2}^{i}+b\left(z_{2}\right)\right) z_{2}^{n} t^{n}+\sum_{i=0}^{n-1} \alpha_{i} t^{i+1} z_{2}^{i+1}=\sum_{i=1}^{\infty} A_{i} t^{i} z_{2}^{i} .
$$

This is equivalent to

$$
A_{i}=\alpha_{i-1} \quad \text { for } i=1, \ldots, n
$$


and for $i=1, \ldots, n$ and $k \geq 0$,

$$
A_{i+(k+1) n}=\frac{1}{t^{(k+1) i+n(1+\ldots+k)}}\left\{\alpha_{i-1}+\sum_{j=0}^{k} b_{i+j n} t^{j i+n(1+\ldots+(j-1))}\right\} .
$$

Then $\Gamma$ is always divergent if not all the coefficients of the formal series vanish. Put

$$
\alpha_{i-1}^{\prime}:=\alpha_{i-1}+\sum_{j=0}^{\infty} b_{i+j n} t^{j i+n(1+\ldots+(j-1))} \quad \text { for } i=1, \ldots, n
$$

where the series is convergent, and

$$
\alpha_{i-1+(k+1) n}^{\prime}=\frac{\alpha_{i-1}^{\prime}}{t^{(k+1) i+n(1+\ldots+k)}} \quad \text { for } 1 \leq i \leq n \text { and } k \geq 0 .
$$

Therefore the sequence $\left(\alpha_{j}^{\prime}\right)$ is exactly such that the sequence of points $\left(O_{j}^{\prime}\right)_{j \geq 0}=\left(\left(\alpha_{j}^{\prime}, 0\right)\right)_{j \geq 0}$ is the sequence of infinitely near points of the blowingups for $F^{\prime}$. If

$$
\begin{gathered}
\beta_{i}=\alpha_{i-1}^{\prime}-\alpha_{i-1}, \quad i=1, \ldots, n, \\
\beta_{i+(k+1) n}:=\alpha_{i-1+(k+1) n}^{\prime}-A_{i+(k+1) n}, \quad i=1, \ldots, n, k \geq 0,
\end{gathered}
$$

and

$$
\varphi(z)=\left(z_{1}+\sum_{j=1}^{\infty} \beta_{j} z_{2}^{j}, z_{2}\right),
$$

then $\varphi$ is an isomorphism of $\mathbb{C}^{2}$ which sends the sequence $\left(O_{j}\right)=\left(\left(A_{j}, 0\right)\right)$ to $\left(O_{j}^{\prime}\right)$. Since $F$ sends $\left(O_{j}\right)$ to itself, $F^{\prime}=\varphi F \varphi^{-1}$ has the expected property and this completes the proof.

Proposition 1.8(iii) and Lemmas 1.12, 1.15 and 1.18 give readily

Theorem 1.19. Any germ

$$
F(z)=\Pi \circ \sigma(z)=\left(\sigma_{1}(z) \sigma_{2}(z)^{n}+\sum_{i=0}^{n-1} \alpha_{i} \sigma_{2}(z)^{i+1}, \sigma_{2}(z)\right)
$$

with $t=\operatorname{tr}(D F(0)) \neq 0$ is conjugate to a normal germ

$$
F^{\prime}(z)=\left(z_{1} z_{2}^{n} t^{n}+\sum_{i=0}^{n-1} \alpha_{i}^{\prime} t^{i+1} z_{2}^{i+1}, t z_{2}\right)
$$

The complement of the rational curves in $\widetilde{S}$ is a Stein manifold, more precisely: 
Corollary 1.20. If $\operatorname{tr}(S) \neq 0$, and $C$ is any rational curve of the universal covering space $\widetilde{S}$ of $S$ then there exists an isomorphism

$$
f: \widehat{S}_{C} \backslash \bigcup_{C^{\prime} \neq C} C^{\prime} \rightarrow \mathbb{C}^{2}
$$

such that the diagram

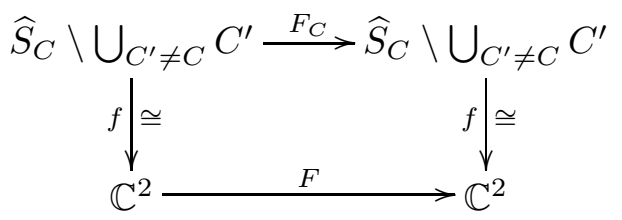

is commutative. In particular $\widetilde{S} \backslash \widetilde{C}$ is isomorphic to $\mathbb{C} \times \mathbb{C}^{\star}$.

Both Theorems 1.4 and 1.19 justify the following definition of the invariant $v(F)$ of germs of mappings $F$. Moreover, it is easy to notice that if we consider the sequence $\left(O_{i}\right)_{i>0}$ of infinitely near points with $O_{i}=\left(\alpha_{i}, 0\right)$ then $\alpha_{i+n}=0$ if and only if $\alpha_{i}=0$. Therefore, given a surface $S$, if we consider other germs $F^{\prime}$ associated with $S$ (for example $F_{C}$ and $F_{C+1}$ in the notations of [D1]), then $v\left(F^{\prime}\right)$ is obtained from $v(F)$ by a cyclic permutation.

Definition 1.21. Let $F^{\prime}=\Pi^{\prime} \sigma^{\prime}$ be a non-zero trace germ and $F$ a normal germ conjugate to $F^{\prime}$. The vanishing invariant $v\left(F^{\prime}\right)=v(F)=$ $\left(\varepsilon_{0}, \ldots, \varepsilon_{n-1}\right)$ is defined by $\varepsilon_{i}=0$ if $\alpha_{i}=0$ and $\varepsilon_{i}=1$ otherwise. The vanishing invariant of a surface $S$ with $\operatorname{tr}(S) \neq 0$ is $v(S)=\left(\varepsilon_{0}, \ldots, \varepsilon_{n-1}\right)$ modulo a cyclic permutation of $\{0,1, \ldots, n-1\}$.

Till now we have only considered the conjugacy relation of germs $F$ : If $F^{\prime}=\varphi^{-1} F \varphi$ then $S(F)$ and $S\left(F^{\prime}\right)$ are isomorphic. In this relation the spherical shell remains fixed. But we may choose another spherical shell and obtain another germ $F^{\prime}$ not conjugate to the previous one but such that $S\left(F^{\prime}\right)$ is nevertheless isomorphic to $S(F)$. We now investigate this situation in order to construct moduli spaces.

LEMMA 1.22. Let $n \geq 1, \mathbb{U}_{n}$ be the group of $n$th roots of unity, $t$ be such that $0<|t|<1, \lambda$ and $\mu$ be such that $\lambda$ and $\mu /|\mu|$ are primitive roots in $\mathbb{U}_{n}$, and $\mu^{n}=t$. Let $G$ be the group of automorphisms of $\mathbb{P}^{n-1}$ generated by

$$
\begin{aligned}
& \Lambda: \mathbb{P}^{n-1} \rightarrow \mathbb{P}^{n-1},\left[\alpha_{0}: \ldots: \alpha_{n-1}\right] \\
& M:\left[\alpha_{0}: \ldots: \lambda^{i} \alpha_{i}: \ldots: \lambda^{n-1} \alpha_{n-1}\right], \\
&: \mathbb{P}^{n-1} \rightarrow \mathbb{P}^{n-1},\left[\alpha_{0}: \ldots: \alpha_{n-1}\right] \mapsto\left[\mu \alpha_{1}: \ldots: \mu^{i} \alpha_{i}: \ldots: \mu^{n-1} \alpha_{n-1}: \alpha_{0}\right] .
\end{aligned}
$$

If $L$ (resp. $T)$ is the group of order $n$ generated by $\Lambda$ (resp. $M)$, then $G$ is a finite commutative group isomorphic to $L \times T$.

Pr o of. Left to the reader. 
TheOREM 1.23. Let

$$
\begin{gathered}
F(z)=\left(z_{1} z_{2}^{n} t^{n}+\sum_{i=0}^{n-1} \alpha_{i} t^{i+1} z_{2}^{i+1}, t z_{2}\right) \quad \text { and } \\
F^{\prime}(z)=\left(z_{1} z_{2}^{n} t^{\prime n}+\sum_{i=0}^{n-1} \alpha_{i}^{\prime} t^{i+1} z_{2}^{i+1}, t^{\prime} z_{2}\right)
\end{gathered}
$$

be normal germs. Then the associated surfaces $S=S(F)$ and $S^{\prime}=S\left(F^{\prime}\right)$ are isomorphic if and only if $t=t^{\prime}$ and $\alpha^{\prime}=\left(\alpha_{0}^{\prime}, \ldots, \alpha_{n-1}^{\prime}\right) \in \mathbb{C}^{n}$ may be obtained from $\alpha=\left(\alpha_{0}, \ldots, \alpha_{n-1}\right) \in \mathbb{C}^{n}$ by a sequence of the following operations:

(i) $\alpha \mapsto \kappa \alpha$ where $\kappa \in \mathbb{C}^{\star}$;

(ii) $\left(\alpha_{0}, \ldots, \alpha_{n-1}\right) \mapsto\left(\alpha_{0}, \lambda \alpha_{1}, \ldots, \lambda^{n-1} \alpha_{n-1}\right)$ where $\lambda^{n}=1$;

(iii) $\left(\alpha_{0}, \ldots, \alpha_{n-1}\right) \mapsto\left(\mu \alpha_{1}, \ldots, \mu^{n-1} \alpha_{n-1}, \alpha_{0}\right)$ where $\mu^{n}=t$.

Proof. By [D1], p. $44, \operatorname{tr}(S)=\operatorname{tr}\left(D F_{C}\left(\widehat{O}_{C}\right)\right)$ for every curve $C$, i.e. the trace does not depend on the choice of a conjugacy class associated with $S$. Let $C$ be a curve in the universal covering space $\widetilde{S}$ of $S$ such that $F=F_{C}$. Then the surfaces $S$ and $S^{\prime}$ are isomorphic if and only if there is an integer $p, 0 \leq p \leq n-1$, such that $F^{\prime}$ and $F_{C+p}$ are conjugate (see [D1], §I.3.11). Therefore by Theorem 1.4, it remains to express $F_{C+1}$ from $F_{C}$ and see how the coefficients are altered.

For this purpose let $F_{0}=F_{C_{0}}$ and $O_{0}=\left(\alpha_{0}, 0\right)$. Then we have the following commutative diagram, where $\Pi_{i}$ is the blowing-up of the curve $C_{i-1}$ at the point $O_{i-1}=\left(\alpha_{i-1}, 0\right)$ :

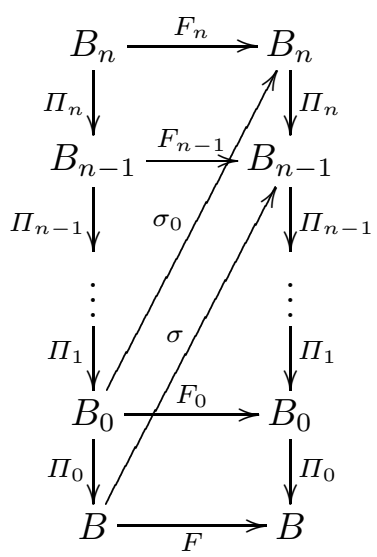

From the relation $\Pi_{0} F_{0}=F \Pi_{0}$, we deduce

$$
F\left(u_{0}, v_{0}\right)=\left(u_{0} v_{0}^{n} t^{n-1}+\sum_{i=0}^{n-1} \alpha_{i} t^{i} v_{0}^{i}, t v_{0}\right) .
$$


By a change of coordinates $U_{0}=u_{0}-\alpha_{0}, V_{0}=v_{0}$ and by conjugation with $\varphi\left(u_{0}, v_{0}\right)=\left(u_{0}, \mu v_{0}\right)$ where $\mu^{n}=t$, it is easy to check that $\left(\alpha_{0}, \ldots, \alpha_{n-1}\right)$ is transformed into $\left(\mu \alpha_{1}, \ldots, \mu^{n-1} \alpha_{n-1}, \alpha_{0}\right)$.

Corollary 1.24. For every $n \geq 1$, there exists an analytic space $\mathcal{M}_{\mathcal{I}}$ (resp. $\left.\mathcal{M}_{\mathcal{G}}\right)$ of all Inoue surfaces (resp. generic surfaces) $S$ for which $b_{2}(S)=n$. More precisely, $\mathcal{M}_{\mathcal{I}}$ (resp. $\left.\mathcal{M}_{\mathcal{G}}\right)$ is isomorphic to $\Delta^{\star}$ (resp. $\left.\mathbb{P}^{n-1} / G \times \Delta^{\star}\right)$.

Pro of. Straightforward by Lemma 1.22 and Theorem 1.23.

The obstruction to constructing a universal family of generic surfaces comes from the existence of non-trivial automorphism groups, therefore we postpone this problem to $\S 3$.

REMARK 1.25. If $F$ is a generic or an Inoue germ which can be defined on $\mathbb{C}^{2}$, then the attraction basin of 0 is an open set $U_{+}=\left\{z \in \mathbb{C}^{2} \mid \exists n\right.$ such that $\left.F^{n}(z) \in B\right\}$ isomorphic to $\mathbb{C}^{2}$ by Corollary 1.20 and "generically" a Fatou-Bieberbach domain, i.e. a domain of $\mathbb{C}^{2}$ isomorphic to $\mathbb{C}^{2}$ but with complement having non-empty interior.

2. Deformations of surfaces containing a GSS. As shown in Section 1 a normal germ of non-zero trace is defined by at most $n+1$ parameters, namely $n$ parameters for the choice of the blown-up points $O_{i}, 1 \leq i \leq n$, on the rational curves, and one parameter for the trace. Moreover if we allow the points $O_{i}$ to move outside the rational curves by $n$ other parameters, we obtain blown-up Hopf surfaces or blown-up surfaces with non-zero trace. In [KH1], F. Kohler proved that these $2 n+1$ parameters give the semi-universal deformation of Inoue surfaces. With similar arguments we shall describe the semi-universal deformation of any surface with non-zero trace.

2.1. Cocycle generators. Let $S=S(\Pi, \sigma)$ be a surface containing a GSS,

$$
\Pi=\Pi_{0} \ldots \Pi_{n-1}: B_{n-1} \rightarrow B_{n-2} \rightarrow \ldots \rightarrow B_{1} \rightarrow B_{0} \rightarrow B
$$

where $\Pi_{i}$ is the blowing-up of $B_{i-1}$ at $O_{i-1} \in C_{i-1}$ and $C_{i}=\Pi_{i}^{-1}\left(O_{i-1}\right)$, with $B_{-1}=B, O_{-1}=0$, and suppose $\sigma: B \rightarrow B_{n-1}$ is an isomorphism onto its image which extends onto a neighbourhood of the closure $\bar{B}$ of $B$. For $i=0, \ldots, n-1$, denote by $A_{i}$ the open set

$$
A_{i}=\Pi_{i}^{-1}\left(\Delta_{i-1}\right) \backslash \Delta_{i}^{\prime} \subset B_{i}
$$

where $\Delta_{i}^{\prime} \subset \subset \Delta_{i}$ are balls in $B_{i}$ centred at $O_{i}, i=0, \ldots, n-1$, and $\Delta_{-1}=B$. The last annulus $A_{n-1}$ is glued to the first one $A_{0}$ thanks to $\sigma \Pi_{0}$. If we still denote by $A_{i}$ the canonical image of $A_{i} \subset B_{i}$ in $S$, the surface $S$ is covered by the family $\mathcal{U}_{E}=\left\{A_{i}\right\}_{i=0, \ldots, n-1}$.

Definition 2.1. The covering $\mathcal{U}_{E}$ is called the Enoki covering of $S$. 
The annulus $A_{i}$ is covered by the two domains $U_{i}$ and $U_{i}^{\prime}$ with coordinate systems respectively $\left(u_{i}, v_{i}\right)$ and $\left(u_{i}^{\prime}, v_{i}^{\prime}\right)$ where $C_{i}=\left\{v_{i}=0\right\}=\left\{v_{i}^{\prime}=0\right\}$, and $A_{i+1}$ and $A_{i}$ are glued along $\Delta_{i} \backslash \overline{\Delta_{i}^{\prime}}$ by

$$
\begin{aligned}
& \Pi_{i+1}\left(u_{i+1}, v_{i+1}\right)=\left(u_{i+1} v_{i+1}+\alpha_{i}, v_{i+1}+\beta_{i}\right), \\
& \Pi_{i+1}\left(u_{i+1}^{\prime}, v_{i+1}^{\prime}\right)=\left(v_{i+1}^{\prime}+\alpha_{i}, u_{i+1}^{\prime} v_{i+1}^{\prime}+\beta_{i}\right),
\end{aligned}
$$

with $O_{i}=\left(\alpha_{i}, \beta_{i}\right), O_{i} \in U_{i}$ or $O_{i} \in U_{i}^{\prime}$. The last gluing between $A_{0}$ and $A_{n-1}$ is given by the mapping

$$
\sigma \Pi_{0}\left(u_{0}, v_{0}\right)=\left(\sigma_{1}\left(u_{0} v_{0}, v_{0}\right)+\alpha_{n-1}, \sigma_{2}\left(u_{0} v_{0}, v_{0}\right)+\beta_{n-1}\right)
$$

or

$$
\sigma \Pi_{0}\left(u_{0}^{\prime}, v_{0}^{\prime}\right)=\left(\sigma_{1}\left(v_{0}^{\prime}, u_{0}^{\prime} v_{0}^{\prime}\right)+\alpha_{n-1}, \sigma_{2}\left(v_{1}^{\prime}, u_{1}^{\prime} v_{1}^{\prime}\right)+\beta_{n-1}\right) .
$$

We are now going to define elements of $H^{1}(\mathcal{U}, \Theta)$ where $\Theta$ is the sheaf of holomorphic tangent fields on $S$. It is well known (see for example [D1]) that if $S$ is not an Inoue surface, then $h^{1}(S, \Theta)=\operatorname{dim}_{\mathbb{C}} H^{1}(S, \Theta)=2 b_{2}(S)$, and $h^{1}(S, \Theta)=2 b_{2}(S)+1$ otherwise. We define cocycles $\theta^{i}$ and $\eta^{i}$ which express respectively the movement of the point $O_{i}$ along the curve $C_{i}$ and outside $C_{i}$ in the following way:

For $i=0, \ldots, n-1$, let $Z^{i}$ be a vector field on the ball $B$ such that:

- $Z^{i}$ vanishes identically on $\sigma^{-1}\left(C_{n-1}\right)$ to order $i+1$,

- for every $0 \leq j \leq i,\left(\Pi_{0} \ldots \Pi_{j}\right)^{\star} Z^{i}$ vanishes identically to order $i-j$ on $C_{j}$ and $\left(1 / v_{j}^{i-j}\right)\left(\Pi_{0} \ldots \Pi_{j}\right)^{\star} Z^{i}$ does not vanish at $O_{j} \in C_{j}$,

- $\left(\Pi_{0} \ldots \Pi_{i}\right)^{\star} Z^{i}$ is tangent to $C_{i}$.

For example, $Z^{i}=z_{2}^{i+1} \partial / \partial z_{1}$ if $\sigma^{-1}\left(C_{n}\right)=\left\{z_{2}=0\right\}$. Since $A_{i} \cap A_{j} \neq \emptyset$ if and only if $i-j= \pm 1 \bmod n$, and $A_{i} \cap A_{i+1}=\Delta_{i} \backslash \overline{\Delta_{i}^{\prime}}$, we define

$$
\theta_{\mid A_{j} \cap A_{k}}^{i}= \begin{cases}\theta_{i, i+1}^{i}=\left(\Pi_{0} \ldots \Pi_{i}\right)^{\star} Z_{\mid A_{i, i+1}}^{i} & \text { for }(j, k)=(i, i+1), \\ \theta_{j, k}^{i}=0 & \text { for }(j, k) \neq(i, i+1) .\end{cases}
$$

By construction, $\theta_{i, i+1}^{i}$ extends to $A_{i}$ and to the previous annuli $A_{0} \cup$ $\ldots \cup A_{i}$ and it vanishes at infinity (i.e. at $C_{i} \cap C_{i-1}$ ). For example, if $F$ is a normal germ, then $\sigma^{-1}\left(C_{n-1}\right)=\left\{z_{2}=0\right\}$ and

$$
\begin{array}{cc}
\theta_{i, i+1}^{i}=\frac{\partial}{\partial u_{i}} & \text { if } O_{i} \in U_{i}, \\
\theta_{\mid A_{j} \cap A_{k}}^{i}=\theta_{j k}^{i}=0 & \text { if }\{j, k\} \neq\{i, i+1\},
\end{array}
$$

satisfies the conditions by Lemma 2.4 (see below).

For $\eta^{i}$, no global conditions are supposed:

$$
\eta_{\mid A_{j} \cap A_{k}}^{i}=\eta_{i, i+1}^{i} \quad \text { for }(j, k)=(i, i+1)
$$


is any vector field transverse to $C_{i}$ such that $\eta_{j k}^{i}\left(O_{i}\right) \neq 0$. For example

$$
\begin{array}{cc}
\eta_{i, i+1}^{i}=\frac{\partial}{\partial v_{i}} & \text { if } O_{i} \in U_{i}, \\
\eta_{i, i+1}^{i}=\frac{\partial}{\partial v_{i}^{\prime}} & \text { if } O_{i} \in U_{i}^{\prime}, \\
\eta_{\mid A_{j} \cap A_{k}}^{i}=\eta_{j k}^{i}=0 & \text { if }\{j, k\} \neq\{i, i+1\} .
\end{array}
$$

Finally, let $T$ be a vector field on $B$ such that:

- $T$ is tangent to $\sigma^{-1}\left(C_{n-1}\right)$ with an isolated zero at 0 , and

- for every $0 \leq i \leq n-1,\left(\Pi_{0} \ldots \Pi_{i}\right)^{\star}(T)$ is tangent to $C_{i}$ with isolated zeros at $O_{i}$ and $C_{i} \cap C_{i-1}$.

Then

$$
\tau_{j k}= \begin{cases}\left(\Pi_{0} \ldots \Pi_{n-1}\right)^{\star}(T) & \text { if }(j, k)=(0, n-1), \\ 0 & \text { if }\{j, k\} \neq\{0, n-1\} .\end{cases}
$$

For example, if $F$ is a normal germ, we notice that the following "trace" cocycle describes the variation of the trace of the surface:

$$
\begin{aligned}
\tau_{j k} & =\left\{\begin{array}{ll}
v_{n-1} \partial / \partial v_{n-1} & \text { on } U_{n-1} \cap \sigma \Pi_{0}\left(A_{0}\right), \\
v_{n-1}^{\prime} \partial / \partial v_{n-1}^{\prime} & \text { on } U_{n-1}^{\prime} \cap \sigma \Pi_{0}\left(A_{0}\right),
\end{array} \quad \text { if }(j, k)=(0, n-1),\right. \\
\tau_{j k} & =0 \quad \text { if }\{j, k\} \neq\{0, n-1\} .
\end{aligned}
$$

It can be easily checked that on $U_{n-1} \cap U_{n-1}^{\prime}, v_{n-1} \partial / \partial v_{n-1}=v_{n-1}^{\prime} \partial / \partial v_{n-1}^{\prime}$ and that this vector field extends to the previous annuli (see Lemma 2.4). All these conditions are invariant under conjugation.

Lemma 2.2. Let $S$ be a minimal surface with a $G S S$ and $n=b_{2}(S)$. If

$$
\sum_{i=0}^{n-1} \lambda_{i}\left[\theta^{i}\right]+\mu_{i}\left[\eta^{i}\right]+\varepsilon[\tau]=0
$$

is a linear relation in $H^{1}(\mathcal{U}, \Theta)$, then $\mu_{i}=0$ for all $i=0, \ldots, n-1$; in particular $\left[\eta^{i}\right] \neq 0$.

Pr o of. Suppose that there exists such a linear relation in $H^{1}(\mathcal{U}, \Theta)$. It means that there exists $X=\left(X_{i}\right) \in C^{0}(\mathcal{U}, \Theta)$ which satisfies

$$
\partial X=\sum_{i=0}^{n-1} \lambda_{i} \theta^{i}+\mu_{i} \eta^{i}+\varepsilon \tau
$$

in $Z^{1}(\mathcal{U}, \Theta)$. This equation is equivalent to the system 


$$
\begin{array}{rlrl}
\left(\sigma \Pi_{0}\right)_{\star} X_{0}-X_{n-1}= & \lambda_{n-1} \theta^{n-1}+\mu_{n-1} \eta^{n-1} & & \\
& +\varepsilon v_{n-1} \frac{\partial}{\partial v_{n-1}} & & \text { on } A_{0} \cap A_{n-1}, \\
\Pi_{n-1 \star} X_{n-1}-X_{n-2}= & \lambda_{n-2} \theta^{n-2}+\mu_{n-2} \eta^{n-2} & & \text { on } A_{n-1} \cap A_{n-2}, \\
\vdots & & \\
\Pi_{1 \star} X_{1}-X_{0}=\lambda_{0} \theta^{0}+\mu_{0} \eta^{0} & & \text { on } A_{1} \cap A_{0} .
\end{array}
$$

The vector field $X_{i}$ extends to the whole blowing-up of the ball $\Delta_{i-1}$ and thus is tangent to the curve $C_{i}$. In particular $\Pi_{i \star} X_{i}\left(O_{i-1}\right)=0$ for $i=$ $1, \ldots, n-1$ and $\left(\sigma \Pi_{0}\right)_{\star} X_{0}\left(O_{n-1}\right)=0$, which gives the result immediately.

The following elementary lemma shows that for generic blowing-ups, it is possible to extend the cocycles to the previous annuli (which is not the case in general).

LEMMA 2.4. For generic blowing-ups, the following holds:

$$
\begin{aligned}
\Pi_{i \star} v_{i}^{j} \frac{\partial}{\partial u_{i}} & =v_{i-1}^{j+1} \frac{\partial}{\partial u_{i-1}}, \\
\Pi_{i \star} u_{i} \frac{\partial}{\partial u_{i}} & =\left(u_{i-1}-\alpha_{i-1}\right) \frac{\partial}{\partial u_{i-1}}, \\
\Pi_{i \star} v_{i}^{k} \frac{\partial}{\partial v_{i}} & =\left(u_{i-1}-\alpha_{i-1}\right) v_{i-1}^{k-1} \frac{\partial}{\partial u_{i-1}}+v_{i-1}^{k} \frac{\partial}{\partial v_{i-1}} .
\end{aligned}
$$

For every $0 \leq i \leq n-1$,

$$
\begin{aligned}
\left(\Pi_{0} \ldots \Pi_{i}\right)_{\star}\left(\frac{\partial}{\partial u_{i}}\right) & =z_{2}^{i+1} \frac{\partial}{\partial z_{1}} \\
\left(\Pi_{0} \ldots \Pi_{n-1}\right)_{\star}\left(v_{n-1} \frac{\partial}{\partial v_{n-1}}\right) & =n z_{1} \frac{\partial}{\partial z_{1}}-\sum_{j=1}^{n-1}(n-j) \alpha_{j-1} z_{2}^{j} \frac{\partial}{\partial z_{1}}+z_{2} \frac{\partial}{\partial z_{2}} .
\end{aligned}
$$

Pro of. Left to the reader.

Lemma 2.5. Let $\Pi: X \rightarrow B$ be the blowing-up of the ball at the origin and $Z$ be a vector field vanishing on the curve $C=\left\{z_{2}=0\right\}$ to order $k>0$. Suppose that the induced foliation $\mathcal{F}$ admits $C$ as invariant curve and the origin is not a singular point of $\mathcal{F}$. Then the induced vector field $\Pi^{\star} Z$ vanishes to order $k-1$ on $C+1=\Pi^{-1}(0)$.

Proof. By assumption

$$
Z(z)=z_{2}^{k}\left(A(z) \frac{\partial}{\partial z_{1}}+z_{2} B(z) \frac{\partial}{\partial z_{2}}\right), \quad A(0) \neq 0 .
$$

If $\left(u_{0}, v_{0}\right)$ is a coordinate system in which $\left(z_{1}, z_{2}\right)=\Pi\left(u_{0}, v_{0}\right)=\left(u_{0} v_{0}, v_{0}\right)$, 
it can be easily checked that

$\Pi^{\star} Z\left(u_{0}, v_{0}\right)$

$$
=v_{0}^{k-1}\left(\left(A\left(u_{0} v_{0}, v_{0}\right)-u_{0} v_{0} B\left(u_{0} v_{0}, v_{0}\right)\right) \frac{\partial}{\partial u_{0}}+v_{0}^{2} B\left(u_{0} v_{0}, v_{0}\right) \frac{\partial}{\partial v_{0}}\right) .
$$

LEMma 2.6. Let $S$ be a minimal surface with a $G S S, n=b_{2}(S) \geq 1$ and $\operatorname{tr}(S) \neq 0$. If

$$
\sum_{i=0}^{n-1} \lambda_{i}\left[\theta^{i}\right]+\varepsilon[\tau]=0
$$

is a linear relation in $H^{1}(\mathcal{U}, \Theta)$, then $\varepsilon=0$. In particular $[\tau] \neq 0$.

Pr o of. We have the following system of equations:

$$
\begin{aligned}
\left(\sigma \Pi_{0}\right)_{\star} X_{0}-X_{n-1} & =\lambda_{n-1} \theta^{n-1}+\varepsilon \tau & & \text { on } A_{0} \cap A_{n-1}, \\
\Pi_{n-1 \star{ }_{\star}} X_{n-1}-X_{n-2} & =\lambda_{n-2} \theta^{n-2} & & \text { on } A_{n-1} \cap A_{n-2}, \\
& \vdots & & \\
\Pi_{1 \star} X_{1}-X_{0} & =\lambda_{0} \theta^{0} & & \text { on } A_{1} \cap A_{0} .
\end{aligned}
$$

By Theorem 1.19, there exists a conjugation of $F=\Pi_{0} \ldots \Pi_{n-1} \sigma$ thanks to which we may suppose that

$$
\begin{gathered}
F\left(z_{1}, z_{2}\right)=\left(z_{1} t^{n} z_{2}^{n}+\sum_{i=0}^{n-1} \alpha_{i} t^{i+1} z_{2}^{i+1}, t z_{2}\right) \text { and } \\
F_{0}\left(u_{0}, v_{0}\right):=F_{C_{0}}\left(u_{0}, v_{0}\right)=\left(u_{0} t^{n-1} v_{0}^{n}+\sum_{i=0}^{n-1} \alpha_{i} t^{i} v_{0}^{i}, t v_{0}\right) .
\end{gathered}
$$

Under this conjugation $\theta^{i}$ and $\tau_{0, n-1}$ are changed into cocycles of the same type. If we write all the equations of (2.7) on $A_{0}$, we obtain

$$
\begin{aligned}
F_{0 \star} X_{0} & \left(F_{0}\left(u_{0}, v_{0}\right)\right)-X_{0}\left(F_{0}\left(u_{0}, v_{0}\right)\right) \\
= & \lambda_{0} \theta^{0}\left(F_{0}\left(u_{0}, v_{0}\right)\right)+\ldots+\lambda_{i}\left(\Pi_{1} \ldots \Pi_{i}\right)_{\star} \theta^{i}\left(F_{0}\left(u_{0}, v_{0}\right)\right)+\ldots \\
& +\lambda_{n-1}\left(\Pi_{1} \ldots \Pi_{n-1}\right)_{\star} \theta^{n-1}\left(F_{0}\left(u_{0}, v_{0}\right)\right) \\
& +\varepsilon\left(\Pi_{1} \ldots \Pi_{n-1}\right)_{\star} \tau\left(F_{0}\left(u_{0}, v_{0}\right)\right) .
\end{aligned}
$$

This means that $F_{0 \star} X_{0}$ extends to $A_{0}$ and thus is tangent to $C_{0}$. Moreover by the first equation of (2.7), $\sigma_{\star} \Pi_{0 \star} X_{0}$ is tangent to $C_{n-1}$, thus $X_{0}$ is tangent to $\Pi_{0}^{-1}\left(\sigma^{-1}\left(C_{n-1}\right)\right)$, in particular it vanishes at infinity. We set $Y=\Pi_{0 \star} X_{0}$; on the ball we have

$$
\left(F_{\star} Y-Y\right)=\sum_{i=0}^{n-1} \lambda_{i}\left(\Pi_{0} \ldots \Pi_{i}\right)_{\star} \theta^{i}+\varepsilon\left(\Pi_{0} \ldots \Pi_{n-1}\right)_{\star} \tau=\sum_{i=0}^{n-1} \lambda_{i} Z_{i}+\varepsilon T .
$$


If $Y(z)=A(z) \partial / \partial z_{1}+z_{2} B(z) \partial / \partial z_{2}$, we have

$$
\begin{aligned}
F_{\star} Y(F(z))-Y(F(z)) & =\left(\begin{array}{cc}
\star & \star \\
0 & t
\end{array}\right)\left(\begin{array}{c}
A(z) \\
z_{2} B(z)
\end{array}\right)-\left(\begin{array}{c}
A(F(z)) \\
t z_{2} B(F(z))
\end{array}\right) \\
& =t z_{2}(B(z)-B(F(z))) \frac{\partial}{\partial z_{2}} \\
& =0 \bmod \left(\frac{\partial}{\partial z_{1}},\left(z_{1}, z_{2}\right)^{2} \frac{\partial}{\partial z_{2}}\right) .
\end{aligned}
$$

By assumption $Z_{0}=z_{2}\left(X(z) \partial / \partial z_{1}+z_{2} Y(z) \partial / \partial z_{2}\right)$; putting this in (2.9) we obtain

$$
\varepsilon T=\varepsilon T+\sum_{i=0}^{n-1} \lambda_{i} Z_{i}=0 \bmod \left(\frac{\partial}{\partial z_{1}},\left(z_{1}, z_{2}\right)^{2} \frac{\partial}{\partial z_{2}}\right)
$$

and $\varepsilon=0$.

2.2. Main example. It is well known that Hopf surfaces $S$ with $H_{1}(S, \mathbb{Z})=$ $\mathbb{Z}$ are obtained by invertible contractions of the type

$$
\gamma\left(z_{1}, z_{2}\right)=\left(a z_{1}+\lambda z_{2}^{m}, b z_{2}\right)
$$

where $m \in \mathbb{N}$ and $a, b, \lambda \in \mathbb{C}$ satisfy the conditions

$$
0<|a| \leq|b|<1 \quad \text { and } \quad\left(b^{m}-a\right) \lambda=0 .
$$

When $\lambda=0, S$ is called a diagonal Hopf surface. The following easy description of global vector fields or elliptic curves of $S$ can be found in [NA] and $[\mathrm{DA}]$.

Lemma 2.10. Let $S$ be a Hopf surface with $H_{1}(S, \mathbb{Z})=\mathbb{Z}$.

(a) If $a \neq b^{m}, m \geq 1$, then there are exactly two elliptic curves $E_{1}=$ $\left\{z_{1}=0\right\}$ and $E_{2}=\left\{z_{2}=0\right\}, h^{0}(S, \Theta)=2$ and global vector fields are given by $\theta(z)=\alpha z_{1} \partial / \partial z_{1}+\beta z_{2} \partial / \partial z_{2}$.

(b) If $a=b^{m}, m \geq 2$ and $\lambda=0$, then $S$ is an elliptic surface, $h^{0}(S, \Theta)=3$ and global vector fields are given by $\theta(z)=\left(\alpha z_{1}+\gamma z_{2}^{m}\right) \partial / \partial z_{1}+$ $\beta z_{2} \partial / \partial z_{2}$.

(c) If $a=b$ and $\lambda=0$, then $h^{0}(S, \Theta)=4, S$ is an elliptic surface and $\theta(z)=\left(\alpha z_{1}+\beta z_{2}\right) \partial / \partial z_{1}+\left(\gamma z_{1}+\delta z_{2}\right) \partial / \partial z_{2}$.

(d) If $\lambda \neq 0$, then there is only one elliptic curve $E=\left\{z_{2}=0\right\}$, $h^{0}(S, \Theta)=2$ and $\theta(z)=\left(\alpha z_{1}+\gamma z_{2}^{m}\right) \partial / \partial z_{1}+\beta z_{2} \partial / \partial z_{2}$ with $\alpha=m \beta$.

Proof. Left to the reader.

Lemma 2.11. Let $S=S(F)$ be a surface containing a GSS, and $F_{u}$ : $B \rightarrow B, z \mapsto F_{u}(z)$, be a deformation of $F(z)=F_{0}(z)$ over $U$ such that $F_{u}(0)=0$ for every $u \in U$. Denote by $\Pi: \mathcal{S} \rightarrow U$ the deformation of $S=S_{0}$ defined by $\left(F_{u}\right)_{u \in U}$. Then for every $u \in U$, the conjugacy class of 
the germ of $F_{u}$ at $0 \in \mathbb{C}^{2}$ defines the minimal surface $S_{u}^{\prime}$ obtained by the blowing-down of the exceptional divisor of $S_{u}=\Pi^{-1}(u)$.

Proof. Without restricting the generality it may be supposed that the mappings $F_{u}$ are "centred" [D1, p. 7] and the proof follows the same lines as the proof of [D1], Lemma 2.7, p. 19.

In the following example which generalizes the explicit versal deformation of Inoue surfaces from [KH1], we compute an explicit versal deformation of all surfaces with non-zero trace, given by the particular cocycles $\eta^{i}=\partial / \partial v_{i}$, $\theta^{i}=\partial / \partial u_{i}$ and $\tau=v_{n-1} \partial / \partial v_{n-1}$. The example corresponds exactly to the movement along or outside the curves $C_{i}$ given by

$$
\Pi_{i}=\left(u_{i}, v_{i}\right)=\left(u_{i} v_{i}+\alpha_{i-1}, v_{i}+\beta_{i-1}\right) \quad \text { for } i=1, \ldots, n-1
$$

and

$$
\begin{aligned}
\sigma \Pi_{0} & =\left(\sigma_{1}\left(u_{0} v_{0}, v_{0}\right)+\alpha_{n-1}, \sigma_{2}\left(u_{0} v_{0}, v_{0}\right)+\beta_{n-1}\right) \\
& =\left(u_{0} v_{0}+\alpha_{n-1}, t v_{0}+\beta_{n-1}\right),
\end{aligned}
$$

$\alpha$ 's, $\beta$ 's and $t$ being parameters of the semi-universal deformation $\mathcal{S} \rightarrow U$. In these explicit examples, $u=(\alpha, \beta, t)$ and $F_{u}$ is obtained by composition of $(*)$ and $(* *)$. More precisely:

THEOREM 2.12. Let $S=S(F)$ be a surface with non-zero trace associated with a normal non-zero trace germ

$$
F(z)=\left(z_{1} z_{2}^{n} t^{n}+\sum_{i=0}^{n-1} \alpha_{i} t^{i+1} z_{2}^{i+1}, t z_{2}\right) .
$$

Then:

(1) If $S$ is an Inoue surface, i.e. if $\alpha_{i}=0$ for every $i$, then the cocycles $\eta^{i}, \theta^{i}$ for $0 \leq i \leq n-1$ and $\tau$ define the semi-universal deformation $\mathcal{S} \rightarrow U$ of $S$. In this deformation the submanifold

$$
M=\left\{\left(\alpha_{0}, \beta_{0}, \ldots, \alpha_{n-1}, \beta_{n-1}, t\right) \mid \beta_{0}=\ldots=\beta_{n-2}=\beta_{n-1}=0\right\}
$$

of codimension $n$ corresponds to minimal surfaces and

$$
\mathcal{I}=\left\{\alpha_{0}=\ldots=\alpha_{n-1}=0\right\}=\left\{z \in U \mid h^{1}\left(S_{z}, \Theta_{z}\right)=2 n+1\right\}
$$

corresponds to blown-up Hopf surfaces or Inoue surfaces at a point on the elliptic curve given by the equation $\left\{z_{1}=0\right\}$. More precisely, there is a flat divisor $\mathcal{E} \subset \mathcal{S}$ over $\mathcal{I}$ such that $E_{u}$ is an elliptic curve of self-intersection $-n$ for every u. Blown-up Hopf surfaces are all diagonal or elliptic (of type (a) or (b) of Lemma 2.10) and $h^{0}\left(S_{u}, \Theta_{u}\right)=1$ for every $u \in \mathcal{I}$.

(2) If $S$ is a generic surface, the cocycles $\eta^{i}, 0 \leq i \leq n-1, \tau$ and $n-1$ among the cocycles $\theta^{i}$ define the semi-universal deformation $\mathcal{S} \rightarrow U$ of $S$, 
if the missing index $j$ is chosen among those for which $\alpha_{j} \neq 0$. In this deformation the submanifold

$$
M=\left\{\left(\alpha_{0}, \beta_{0}, \ldots, \widehat{\alpha}_{j}, \beta_{j}, \ldots, \alpha_{n-1}, \beta_{n-1}\right) \mid \beta_{0}=\ldots=\beta_{n-1}=0\right\}
$$

of codimension $n$ corresponds to minimal surfaces and if $u \notin M$, then $S_{u}$ is a blown-up generic surface or a blown-up Hopf surface.

(3) In both cases there exists a flat divisor $\mathcal{C}$ over $U$ such that $C_{u}$ is an elliptic curve if $S_{u}$ is a blown-up Hopf surface, and a cycle of rational curves in all other cases. In all cases $C_{u}^{2}=0$.

Pr o o f. (a) We recall that $h^{1}(S, \Theta)=2 b_{2}(S)+h^{0}(S, \Theta)$. By the KodairaSpencer theorem, it is sufficient to show that these cocycles are linearly independent. According to the above Lemma 2.2, we only have to prove the linear independence of the cocycles $\theta^{i}$ and $\tau$ in the first case, and of $n-1$ among the $\theta$ 's and $\tau$ in the second. Suppose there exists a linear relation; hence there exists $X=\left(X_{i}\right) \in C^{0}(\mathcal{U}, \Theta)$ such that

$$
\partial X=\sum_{i=0}^{n-1} \lambda_{i} \theta\left(\alpha_{i}\right)+\varepsilon \tau .
$$

By Lemma 2.6, $\varepsilon=0$ and we have the system

$$
\begin{aligned}
\left(\sigma \Pi_{0}\right)_{\star} X_{0}-X_{n-1} & =\lambda_{n-1} \frac{\partial}{\partial u_{n-1}} & & \text { on } A_{0} \cap A_{n-1}, \\
\Pi_{n-1 \star} X_{n-1}-X_{n-2} & =\lambda_{n-2} \frac{\partial}{\partial u_{n-2}} & & \text { on } A_{n-1} \cap A_{n-2}, \\
& \vdots & & \\
\Pi_{1 \star} X_{1}-X_{0} & =\lambda_{0} \frac{\partial}{\partial u_{0}} & & \text { on } A_{1} \cap A_{0} .
\end{aligned}
$$

These equations are in fact valid on the whole annulus since $\partial / \partial u_{i}, u_{i} \partial / \partial u_{i}$ and $v_{n-1} \partial / \partial v_{n-1}$ extend to the annulus $A_{i-1}$ :

$$
\begin{array}{rlrl}
\left(\sigma \Pi_{0}\right)_{\star} X_{0}-X_{n-1} & =\lambda_{n-1} \frac{\partial}{\partial u_{n-1}} & & \text { on } A_{n-1}, \\
\Pi_{n-1_{\star}} X_{n-1}-X_{n-2} & =\lambda_{n-2} \frac{\partial}{\partial u_{n-2}} & & \text { on } A_{n-2}, \\
& \vdots & \\
\Pi_{1 \star} X_{1}-X_{0} & =\lambda_{0} \frac{\partial}{\partial u_{0}} & & \text { on } A_{0} .
\end{array}
$$

By induction and according to Lemma 2.4 we send all the equations of (2.13) 
down to $A_{0}$. We prove by induction on $k \geq 0$ that for some constants $\lambda_{i}^{\prime}$, $\left(\Pi_{n-k} \ldots \Pi_{n-1} \sigma \Pi_{0}\right)_{\star} X_{0}=X_{n-k-1}+\left(\lambda_{n-1}^{\prime} v_{n-k-1}^{k}+\ldots+\lambda_{n-k-1}^{\prime}\right) \frac{\partial}{\partial u_{n-k-1}}$.

For $k=n-1$ this yields

$$
F_{\star} X_{0}\left(F\left(u_{0}, v_{0}\right)\right)-X_{0}\left(F\left(u_{0}, v_{0}\right)\right)=\left.\left(\sum_{i=0}^{n-1} \lambda_{i}^{\prime} v_{0}^{i}\right) \frac{\partial}{\partial u_{0}}\right|_{F\left(u_{0}, v_{0}\right)}
$$

where

$$
F\left(u_{0}, v_{0}\right):=\Pi_{1} \ldots \Pi_{n-1} \sigma \Pi_{0}\left(u_{0}, v_{0}\right)=\left(u_{0} v_{0}^{n} t^{n-1}+\sum_{i=0}^{n-1} \alpha_{i} t^{i} v_{0}^{i}, t v_{0}\right) .
$$

The associated jacobian matrix is

$$
D F\left(u_{0}, v_{0}\right)=\left(\begin{array}{cc}
v_{0}^{n} t^{n-1} & n u_{0} v_{0}^{n-1} t^{n-1}+\sum_{i=1}^{n-1} \alpha_{i} i t^{i} v_{0}^{i-1} \\
0 & t
\end{array}\right) .
$$

Since $X_{0}$ is tangent to $C_{0}$ we write

$$
X_{0}\left(u_{0}, v_{0}\right)=A\left(u_{0}, v_{0}\right) \frac{\partial}{\partial u_{0}}+v_{0} B\left(u_{0}, v_{0}\right) \frac{\partial}{\partial v_{0}} .
$$

Putting this in (2.14) we obtain immediately

$$
B\left(u_{0}, v_{0}\right)-B\left(F\left(u_{0}, v_{0}\right)\right)=0 .
$$

The latter equation means that $B$ defines a global holomorphic function on $S$ which is compact. Thus $B$ is a constant and (2.14) is equivalent to

$v_{0}^{n} t^{n-1} A\left(u_{0}, v_{0}\right)+\left(n u_{0} v_{0}^{n} t^{n-1}+\sum_{i=1}^{n-1} \alpha_{i} i t^{i} v_{0}^{i}\right) B-A\left(F\left(u_{0}, v_{0}\right)\right)=\sum_{i=0}^{n-1} \lambda_{i} t^{i} v_{0}^{i}$

Comparing the terms containing $u v^{n}$, we obtain $B=0$ and (2.14) becomes

$$
v_{0}^{n} t^{n-1} A\left(u_{0}, v_{0}\right)-A\left(F\left(u_{0}, v_{0}\right)\right)=\sum_{i=0}^{n-1} \lambda_{i} t^{i} v_{0}^{i} .
$$

Differentiation of (2.15) with respect to $u$ yields

$$
\frac{\partial A}{\partial u}\left(u_{0}, v_{0}\right)=\frac{\partial A}{\partial u}\left(F\left(u_{0}, v_{0}\right)\right) .
$$

As before, this shows that $(\partial A / \partial u)\left(u_{0}, v_{0}\right)$ is constant, i.e. $A\left(u_{0}, v_{0}\right)=a u_{0}+$ $V\left(v_{0}\right)$ where $V$ satisfies the condition

$$
v_{0}^{n} t^{n-1} V\left(v_{0}\right)-V\left(t v_{0}\right)=\sum_{i=0}^{n-1}\left(a \alpha_{i}+\lambda_{i}\right) t^{i} v_{0}^{i} .
$$

We claim that

$$
a \alpha_{i}+\lambda_{i}=0, \quad i=0, \ldots, n-1,
$$


and $V=0$. To see this, set $V\left(v_{0}\right)=\sum_{i=0}^{\infty} b_{i} v_{0}^{i}$. By (2.16),

$$
\begin{aligned}
b_{i} & =-\left(a \alpha_{i}+\lambda_{i}\right) \quad \text { for } i=0, \ldots, n-1, \\
b_{n+j} & =b_{j} / t^{j+1} .
\end{aligned}
$$

For $j \in \mathbb{N}$, euclidean division gives $j=k n+i$ with $i \in\{0, \ldots, n-1\}$. By induction on $k \geq 0$,

$$
b_{j}=\frac{b_{i}}{t^{n(k-1) k / 2+k(i+1)}} .
$$

Suppose that there exists an index $i$ such that $a \alpha_{i}+\lambda_{i} \neq 0$. The corresponding subseries $\sum b_{q n+i} t^{q n+i}$ is divergent. In fact

$$
\frac{b_{q n+n+i} t^{q n+n+i}}{b_{q n+i} t^{q n+i}}=\frac{t^{n}}{t^{n q+i+1}}=\frac{1}{t^{n(q-1)+i+1}} .
$$

Hence $V$ is divergent outside the ball of radius $|t|$. Nevertheless $X_{0}$ is defined on $A_{0}$, in particular it is convergent on $\left\{\left.\left(u_{0}, v_{0}\right)|| u_{0} v_{0}\right|^{2}+\left|v_{0}\right|^{2}<1\right\}$. On $\left\{u_{0}=0\right\}$, the vector field $X_{0}\left(0, v_{0}\right)=V\left(v_{0}\right) \partial / \partial u_{0}$ should converge on the unit ball, which is impossible as has just been seen.

Finally, $X_{0}=a u_{0} \partial / \partial u_{0}$. By induction we obtain $X_{i}=a u_{i} \partial / \partial u_{i}$ and there is a linear relation

$$
\left(\lambda_{0}, \ldots, \lambda_{n-1}\right)=-a\left(\alpha_{0}, \ldots, \alpha_{n-1}\right) \quad \text { for } a \in \mathbb{C} .
$$

This is the trivial linear relation if and only if $\alpha_{i}=0$ for all $i$ and in this case the equations show that there is a global vector field, i.e. $S$ is an Inoue surface.

Moreover, if $\alpha_{j}=0$, then $\lambda_{j}=0$ necessarily.

If $\alpha_{j} \neq 0$ and $\lambda_{j}=0$, then $a=0$ and all $\lambda_{i}$ vanish. Therefore the missing index has to be chosen among those for which $\alpha_{j} \neq 0$, because in this case $\lambda_{j} \neq 0$ and $\theta_{j}$ can be expressed through the other $\theta_{i}$.

(b) The fact that $M$ corresponds to minimal surfaces is clear. The vanishing of $\alpha_{i}$ gives rise to the mapping

$$
F\left(z_{1}, z_{2}\right)=\left(z_{1} \prod_{i=0}^{n-1}\left(t z_{2}+\beta_{n-1}+\ldots+\beta_{i}\right), t z_{2}+\beta_{n-1}+\ldots+\beta_{0}\right)
$$

or

$$
F\left(z_{1}, z_{2}\right)=\left(z_{1} \prod_{i=0}^{n-1}\left(t z_{2}+\beta_{n-1}+\ldots+\beta_{i}\right), t z_{2}+\beta_{n-1}+\ldots+\beta_{0}+\beta_{-1}\right)
$$

with $\sum_{i=-1}^{n-1} \beta_{i}=0$ if we want the condition $F(0)=0$. The blown-up points are on the strict transform of the line $E=\left\{z_{1}=0\right\}$ which gives an elliptic curve $C_{0 \beta t}$ in the surface $S_{0 \beta t}$. Moreover $S_{0 \beta t}$ is a blown-up Hopf surface if all $\beta_{n-1}+\ldots+\beta_{i} \neq 0$, and a blown-up Inoue surface if there is at least one index $i$ for which $\beta_{n-1}+\ldots+\beta_{i}=0$. Clearly $z_{1} \partial / \partial z_{1}$ induces a global vector 
field on $S_{0 \beta t}$. The equation $\left\{z_{2}=0\right\}$ is defined for the family of germs $F_{u}$ for every $u \in U$; if $S_{u}$ is a blown-up Hopf surface, the contraction $z_{1} \mapsto a z_{1}$ with small $a \neq 0$, computed from $\alpha_{i}$ and $\beta_{i}$, gives an elliptic curve; if $S_{u}$ is obtained by a blowing-up of a non-zero trace surface with $b_{2}>0$ it gives exactly the cycle of $b_{2}$ rational curves by Lemma 2.11 . This completes the proof.

2.3. General case

THEOREM 2.19. Let $S=S(F)=S(\Pi, \sigma)$ be a minimal surface containing a GSS such that $n=b_{2}(S) \geq 1$ and $\operatorname{tr}(S) \neq 0$. Then the cocycles $\eta^{i}, \theta^{i}$ for $i=0, \ldots, n-1$ and $\tau$ are generators of $H^{1}(S, \Theta)$. More precisely, let $v(F)=\left(\varepsilon_{0}, \ldots, \varepsilon_{n-1}\right)$ be the vanishing invariant of $F$.

(i) If $h^{1}(S, \Theta)=2 n+1$ then the cocycles $\eta^{i}, \theta^{i}$ for $i=0, \ldots, n-1$ and $\tau$ are a base of $H^{1}(S, \Theta)$.

(ii) If $h^{1}(S, \Theta)=2 n$ and $j$ is an index such that $\varepsilon_{j}=1$ then the cocycles $\eta^{i}$ for $i=0, \ldots, n-1, \theta^{i}$ for $i \neq j$, and $\tau$ are a base of $H^{1}(S, \Theta)$.

P r o of. By Theorem 1.19 it may be supposed that $F$ is a normal germ. By Lemmas 2.2 and 2.6 it is sufficient to prove that $\left[\theta^{i}\right]$ are linearly independent in case (i), and in case (ii) that $\left[\theta^{i}\right]$ are linearly independent for $i \neq j$ such that $\varepsilon_{j}=1$. Let $\mathcal{S} \rightarrow B$ be the versal deformation given in Theorem 2.12.

(i) By Theorem 1.4, if $\sum \lambda_{i} \theta^{i} \neq 0$, then the associated deformation $\mathcal{T} \rightarrow U$, which exists since $H^{2}(S, \Theta)=0$, is not locally trivial, moreover the coordinates of the blown-up points are obtained by integration of the cocycles, therefore the base change mapping $H:(U, 0) \rightarrow(B, 0)$ is invertible and $\sum \lambda_{i}\left[\theta^{i}\right] \neq 0$.

(ii) By Theorem 1.4, if $\sum \lambda_{i} \theta^{i} \neq 0$, there is a relation $\sum \lambda_{i}\left[\theta^{i}\right]=0$ only if integration of the vector fields $\theta^{i}$ moves the points $O_{i}=\left(\alpha_{i}, 0\right)$ in such a way that $\alpha_{i}(u)=\kappa(u) \alpha_{i}$ for every $i$. But this is impossible if we have removed $\theta^{j}$ such that $\alpha_{j} \neq 0$.

Corollary 2.20. Let $S=S(F)$ be a surface with $\operatorname{tr}(S) \neq 0$. Then every deformation $\mathcal{S} \rightarrow Y$ of $S$ where $S \simeq S_{0}$ is locally given by a deformation of any $F^{\prime}$ such that $F \sim F^{\prime}$. More precisely, for any choice of a representative $F^{\prime}$ in the conjugacy class of $F$ and any choice of charts, there exists a neighbourhood $Y^{\prime} \subset Y$ of 0 and holomorphic functions $\alpha_{0}(y), \ldots, \alpha_{n-1}(y)$, $\beta_{0}(y), \ldots, \beta_{n-1}(y)$ and $t(y)$ on $Y^{\prime}$ such that if

$$
F^{\prime}(z, y)=\Pi_{0}^{\prime} \ldots \Pi_{n-1}^{\prime} \sigma^{\prime}(z, y)
$$

with

$$
\Pi_{i}^{\prime}\left(u_{i}, v_{i}, y\right)=\left(u_{i} v_{i}+\alpha_{i-1}(y), v_{i}+\beta_{i-1}(y)\right)
$$

or 


$$
\Pi_{i}^{\prime}\left(u_{i}^{\prime}, v_{i}^{\prime}, y\right)=\left(v_{i}^{\prime}+\alpha_{i-1}(y), u_{i}^{\prime} v_{i}^{\prime}+\beta_{i-1}(y)\right)
$$

for $i=1, \ldots, n-1$,

$$
\Pi_{0}^{\prime}\left(u_{0}, v_{0}\right)=\left(u_{0} v_{0}, v_{0}\right) \quad \text { or } \quad \Pi_{0}^{\prime}\left(u_{0}^{\prime}, v_{0}^{\prime}\right)=\left(v_{0}^{\prime}, u_{0}^{\prime} v_{0}^{\prime}\right)
$$

and

$$
\sigma(z, y)=\left(z_{1}+\alpha_{n-1}(y), t(y) z_{2}+\beta_{n-1}\right)
$$

then $S_{y} \simeq S\left(F^{\prime}(\cdot, y)\right)$ provided the point $O_{i}$ belongs to the two domains of charts.

Pr o of. Comes from the definition of a versal deformation.

Corollary 2.21. Let $S$ have $\operatorname{tr}(S) \neq 0$. Then the unique holomorphic foliation extends to any deformation of $S$.

Proof. Any deformation is given by a deformation $F_{u}=\left(\star, t(u) z_{2}+\right.$ $\left.\beta_{0}(u)+\ldots+\beta_{n-1}(u)\right)$.

3. Automorphisms. Let $\operatorname{Aut}(S)$ be the group of automorphisms of $S$; $\operatorname{Aut}(S)$ is a complex Lie group by the theorem of Bochner-Montgomery. Let $D_{i}, 0 \leq i \leq n-1$, be the $n$ rational curves of $S$. Following [D3] we denote by $\operatorname{Aut}_{0}(S)$ the normal closed subgroup of automorphisms $g$ with $g\left(D_{i}\right)=D_{i}$ for every $0 \leq i \leq n-1$. By Lemma 3.4 of [D3], this group is isomorphic to the group $\operatorname{Isom}\left(F_{C}\right)$ of invertible germs $\varphi$ such that $\varphi F_{C}=F_{C} \varphi$. In the same way, if $p$ is a divisor of $n$, then $\operatorname{Aut}_{p}(S)$ is the set of automorphisms $g: S \rightarrow S$ which have no fixed points and satisfy $g\left(D_{i}\right)=D_{i+p}$. By Theorems 1.4 and 1.19 we have immediately

TheOrem 3.1. Let $S$ be a minimal surface with $n=b_{2}(S)$ and $\operatorname{tr}(S) \neq 0$.

(1) If $S$ is an Inoue surface, i.e. $v(F)=(0, \ldots, 0)$, then $\operatorname{Aut}_{0}(S) \simeq$ $\mathbb{C}^{\star} \times \mathbb{Z} / n \mathbb{Z}$ and $\mathbb{C}^{\star}$ corresponds to the one-parameter group generated by a non-zero global vector field.

(2) If $v(S)$ contains exactly one non-zero coefficient, then $\operatorname{Aut}_{0}(S) \simeq$ $\mathbb{Z} / n \mathbb{Z}$.

(3) If $v(S)$ has at least two non-zero coefficients then $\operatorname{Aut}_{0}(S) \simeq$ $\mathbb{Z} / \operatorname{gcd}\{m, n\} \mathbb{Z}$, where $1 \leq m \leq n-1$ is the least integer such that $m=$ $k-j \bmod n$, with $\varepsilon_{j}=1$ and $\varepsilon_{k}=1$. In particular, generically $\operatorname{Aut}_{0}(S)$ $=\{\operatorname{Id}\}$.

It is easy to see that any Inoue surface with $b_{2}(S)=n$ is an $n$-fold covering of an Inoue surface with $b_{2}(S)=1$; nevertheless generically a surface with a GSS and $b_{2}(S)>1$ is not a covering of another surface, as we are going to see in the sequel. 
Proposition 3.2. Let $S$ be a generic surface with $n=b_{2}(S) \geq 1$.

(1) Let $\mathcal{S} \rightarrow U$ be the versal logarithmic deformation of $S \simeq S_{0}$, where $\operatorname{dim} U=n$. Let $p, q \in \mathbb{N}^{\star}$ be such that $n=p q$, and $X_{q} \subset U$ be the subset of points $u$ such that $S_{u}$ is a q-fold covering of another generic surface. Then $X_{q}$ is a submanifold of $U$ of dimension $p$.

(2) If $\operatorname{Aut}_{p}(S) \neq \emptyset$, then there exists $\alpha \in \operatorname{Aut}_{p}(S)$ such that $\alpha^{q}=\mathrm{Id}$. In this case and for $p>0$ minimal with this property, denote by $G \simeq \mathbb{Z} / q \mathbb{Z}$ the cyclic group generated by $\alpha$. Then $\operatorname{Aut}(S)$ is a finite commutative group isomorphic to the product $\operatorname{Aut}_{0}(S) \times G$ of cyclic groups.

Proof. (1) Let $F_{C}$ be the germ associated with the curve $C$ at the point $\widehat{O}_{C}=\left(\alpha_{0}, 0\right)$. We have $F_{C}(u, v)=\left(u v^{n} t^{n-1}+\sum_{i=0}^{n-1} \alpha_{i} v^{i} t^{i}, t v\right)$ and with the notations of [D1], $F_{C+p}$ satisfies the commutative diagram

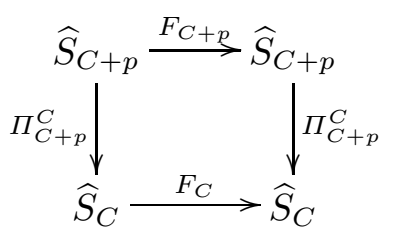

where $\Pi_{C+p}^{C}(u, v)=\left(u v^{p}+\sum_{i=0}^{p-1} \alpha_{i} v^{i}, v\right)$. A straightforward computation gives $F_{C+p}(u, v)=\left(u v^{n} t^{n-p-1}+\sum_{i=0}^{n-1} \alpha_{i}^{\prime} t^{i} v^{i}, t v\right)$ with

$$
\begin{aligned}
\left(\alpha_{0}^{\prime}, \ldots, \alpha_{n-p-1}^{\prime}, \alpha_{n-p}^{\prime}, \ldots, \alpha_{n-1}^{\prime}\right) & \\
& =\left(\alpha_{p}, \ldots, \alpha_{n-1}, \alpha_{0} / t, \alpha_{1} / t^{2}, \ldots, \alpha_{p-1} / t^{p}\right) .
\end{aligned}
$$

Now any $\alpha \in \operatorname{Aut}_{p}(S)$ induces, for every curve $C$, an isomorphism $\widehat{\alpha}_{C}$ such that

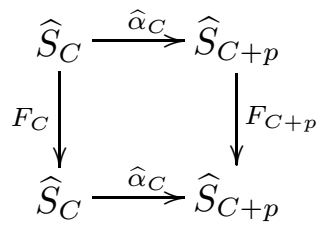

is commutative. By Theorem 1.4(1), there exist $\kappa$ and $\mu$ such that

$$
\widehat{\alpha}_{C}(u, v)=\left(\kappa\left(u-\alpha_{0}\right)+\alpha_{p}, \mu v\right),
$$

therefore $t^{p}=\mu^{n}$ and

$$
\begin{array}{cc}
\mu^{q}=e^{2 i \pi m / p} t & \text { for } 0 \leq m \leq p-1, \\
\alpha_{j}^{\prime} \mu^{j}=\kappa \alpha_{j} & \text { for } 0 \leq j \leq n-1 .
\end{array}
$$

Conversely, for any $m$, such an $\widehat{\alpha}_{C}$ defines an automorphism of $\operatorname{Aut}_{p}(S)$. 
The linear system given by (3.3) and (3.6) splits into $p$ linear systems

$$
\begin{aligned}
\alpha_{p+j} \mu^{j} & =\kappa \alpha_{j}, \\
\alpha_{2 p+j} \mu^{p+j} & =\kappa \alpha_{p+j}, \\
& \vdots \\
\alpha_{(q-1) p+j} \mu^{(q-2) p+j} & =\kappa \alpha_{(q-2) p+j}, \\
\frac{\alpha_{j}}{t^{j+1}} \mu^{(q-1) p+j} & =\kappa \alpha_{(q-1) p+j},
\end{aligned}
$$

for $j=0, \ldots, p-1$. $\left(3.7_{j}\right)$ shows that for every $0 \leq j \leq p-1$, all $\alpha_{k p+j}=0$ or $e^{2 i \pi m j / p} \mu^{p q(q-1) / 2}=t \kappa^{q}$. Finally, we have $p$ parameters $\alpha_{0}, \ldots, \alpha_{p-1}$ which determine linearly the other $\alpha_{j}$, and define a (disconnected) manifold $X_{q}^{\prime}$ of dimension $p$. Since these conditions are necessary we have $X_{q} \subset$ $X_{q}^{\prime} \subset U$. The next step is to show that if $\operatorname{Aut}_{p}(S) \neq \emptyset$ then there exists an automorphism $\alpha \in \operatorname{Aut}_{p}(S)$ such that $\alpha^{q}=\mathrm{Id}$. To see this, suppose that $m=0$. Then by $(3.5)$ and $\left(3.7_{j}\right)$,

$$
\mu^{q}=t \quad \text { and } \quad \mu^{p q(q-1) / 2}=t \kappa^{q} .
$$

From $\left(3.7_{j}\right)$ we deduce

$$
\alpha_{l p+j}=\frac{\kappa^{l} \alpha_{j}}{\mu^{l j+p l(l-1) / 2}} \quad \text { for } 0 \leq j \leq p-1 \text { and } 0 \leq l \leq q-1 .
$$

Define $G_{C}$ by

$$
G_{C}(u, v)=\Pi_{C+p}^{C} \widehat{\alpha}_{C}(u, v)=\left(\kappa u \mu^{p} v^{p}+\sum_{i=0}^{p-1} \alpha_{i} \mu^{i} v^{i}, \mu v\right) .
$$

By [D3], Lemma 3.6, it is sufficient to prove that $G_{C}^{q}=F_{C}$. By induction on $k>0$ it is easy to check that

$$
\begin{aligned}
G_{C}^{k}(u, v)= & \left(\kappa^{k} u v^{k p} \mu^{p(1+\ldots+k)}+\kappa^{k-1} \sum_{i=0}^{p-1} \alpha_{i} \mu^{i+(2+\ldots+k) p} v^{i+(k-1) p}+\ldots\right. \\
& \left.+\kappa^{l} \sum_{i=0}^{p-1} \alpha_{i} \mu^{(k-l) i+((k-l+1)+\ldots+k) p} v^{i+l p}+\ldots+\sum_{i=0}^{p-1} \alpha_{i} \mu^{k i} v^{i}, \mu^{k} v\right) .
\end{aligned}
$$

For $k=q$ we have the result and $X_{q}=X_{q}^{\prime}$ by [D3], 3.8, p. 681 .

(2) As has already been seen, if $p$ is the least integer such that $\operatorname{Aut}_{p}(S)$ $\neq \emptyset$ then there exists $\alpha$ such that $\alpha^{q}=$ Id. Moreover if $\beta \in \operatorname{Aut}_{p^{\prime}}(S)$ then there exist $q^{\prime}$ and $\varphi \in \operatorname{Aut}_{0}(S)$ which satisfy $p^{\prime}=q^{\prime} p$ and $\beta=\alpha^{q^{\prime}} \varphi$. Therefore in order to prove the commutativity of $\operatorname{Aut}(S)$ it is sufficient to 
check that for a given curve $C$ the diagram

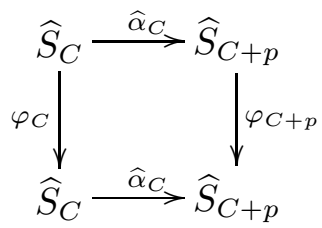

commutes. If all $\alpha_{i}=0$, the result is clear, thus we may suppose that $\alpha_{0} \neq 0$; but then $\alpha_{p} \neq 0$, thus by Theorem 1.4(2) we have $\lambda^{\operatorname{gcd}(p, n)}=1$ and $\varphi_{C}(u, v)=(u, \lambda v)$. Moreover, by $(3.4)$ and $\left(3.7_{0}\right), \widehat{\alpha}_{C}(u, v)=(\kappa u, \mu v)$. To complete the proof we have to compute $\widehat{\varphi}_{C+p}$ from the commutativity of

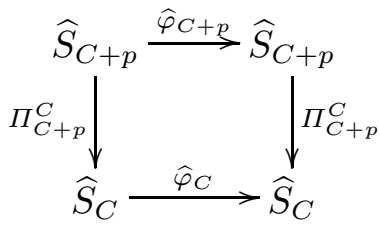

with $\Pi_{C+p}^{C}(u, v)=\left(u v^{p}+\sum_{i=0}^{p-1} \alpha_{i} v^{i}, v\right)$. Note that by Theorem 1.4, $\lambda^{\operatorname{gcd}\{m, n\}}$ $=1$, thus $\sum \alpha_{i} v^{i}=\sum \alpha_{i} \lambda^{i} v^{i}$, so $\widehat{\varphi}_{C+p}(u, v)=\left(u / \lambda^{p}, \lambda v\right)=(u, \lambda v)$ and the result is obvious.

Lemma 3.9. Let $\mathcal{S} \rightarrow U$ be a family of generic surfaces with $n=b_{2}\left(S_{u}\right)$ $>0$, where $U$ is connected and $n=p q$. If there is an open subset $V \subset U$ such that for every $u \in V, S_{u}$ is a q-fold covering of a surface $S_{u}^{\prime}$, then every surface is a q-fold covering surface. Moreover if $U$ is simply connected, then there exists a family $\mathcal{S}^{\prime} \rightarrow U$ of generic surfaces with $p=b_{2}\left(S_{u}^{\prime}\right)$ and a commutative diagram

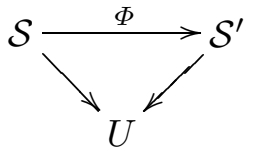

such that for every $u \in U,\left(S_{u}, \Phi_{u}, S_{u}^{\prime}\right)$ is a q-fold covering.

Proof. By Proposition 3.2(1), the set of points $u$ such that $S_{u}$ is a $q$-fold covering is an analytic subset; since it contains $V$, it contains $U$. By Corollary 2.21 the trace depends holomorphically on $u$ and conditions (3.8) become

$$
\mu(u)^{q}=t(u) \quad \text { and } \quad \mu(u)^{p q(q-1) / 2}=\kappa(u)^{q} .
$$

By choosing a determination of $z^{1 / q}, \kappa(u)$ and $\mu(u)$ may be defined on $U$, therefore an automorphism $g$ is globally defined on $\mathcal{S}$. For every $u \in U$, $g_{u} \in \operatorname{Aut}_{p}\left(S_{u}\right)$ satisfies $g_{u}^{q}=\operatorname{Id}_{S_{u}}$. If $\mathcal{G}$ denotes the group generated by $g$, then $\mathcal{S}^{\prime}=\mathcal{S} / \mathcal{G}$ and $\Phi$ is the canonical mapping $\Phi: \mathcal{S} \rightarrow \mathcal{S}^{\prime}$. 
Definition 3.10. Let $D$ be a rational curve of a surface $S$ with a GSS. Then $(S, D)$ will be called a marked surface. A deformation of a marked surface $(S, D)$ is a 6 -uple $\left(\mathcal{S}, \theta, U, \mathcal{D}, f, u_{0}\right)$ which satisfies the following conditions:

(i) $f: S \rightarrow S_{u_{0}}$ is an isomorphism;

(ii) $\theta: \mathcal{S} \rightarrow U$ is a deformation of $S_{u_{0}}$;

(iii) $\mathcal{D}$ is a divisor of $\mathcal{S}$ flat over $U$ such that $f_{\star}(D)=D_{u_{0}}$.

Similarly, the 4-uple $(\mathcal{S}, \theta, U, \mathcal{D})$ will be called a family of marked surfaces.

We recall that if $D$ is an effective divisor on $S$, then the locally free sheaf $\Omega_{S}^{1}(\log D)$ defined by

$$
\Omega_{S}^{1}(\log D)(U):=\left\{\omega \in \Omega_{S}^{1}\left(D_{\text {red }}\right)(U) \mid d \omega \in \Omega_{S}^{2}\left(D_{\text {red }}\right)(U)\right\}
$$

is called the sheaf of meromorphic forms with logarithmic poles in $D$. If $z \in D$ is a regular point and $D=\left\{z_{1}=0\right\}$, then $\Omega_{S}^{1}(\log D)$ is generated by $d z_{1} / z_{1}$ and $d z_{2}$; if $z \in D$ is a singular point and $D=\left\{z_{1} z_{2}=0\right\}$ then $\Omega_{S}^{1}(\log D)$ is generated by $d z_{1} / z_{1}$ and $d z_{2} / z_{2}$. A logarithmic deformation [KW] is defined by cocycles in the dual sheaf $\Theta_{S}(-\log D):=\mathcal{H}_{\mathcal{O}_{S}}\left(\Omega_{S}^{1}(\log D), \mathcal{O}_{S}\right)$, therefore configuration of curves is maintained. Given a structure on manifolds we shall say that there is a logarithmic fine moduli space if the set $\mathcal{X}$ of isomorphism classes can be endowed with a structure of analytic space and there is a family $\theta: \mathcal{U} \rightarrow \mathcal{X}$ which is the universal logarithmic deformation at every point. With the notations of Lemma 1.22, we have

TheOREM 3.11. There is a logarithmic fine moduli space of all marked generic surfaces $S$ with $b_{2}(S)=n \geq 1$ and it is isomorphic to $\mathbb{P}^{n-1} / L \times \Delta^{\star}$.

Pro of. The group $L$ acts on $\mathbb{P}^{n-1}$ and has $n$ fixed points $\left[0: \ldots: 0: \alpha_{i}\right.$ : $0: \ldots: 0]$ which give in the quotient $n$ normal isolated singularities. Let $U$ be the complement of these singular points. The set $U^{\prime}$ of points $u \in U$ such that $\operatorname{Aut}\left(S_{u}\right)=\{\operatorname{Id}\}$ is Zariski dense by Theorem 3.1 and Proposition 3.2(1). For every $u \in U$ we choose a representative of the versal logarithmic deformation $\mathcal{S}_{u} \rightarrow V_{u}$ over a small neighbourhood $V_{u}$ of $u$ such that over $V_{u}$ any two surfaces are not isomorphic; that is possible because $L$ is finite and we have removed the fixed points. Over $V_{u} \cap V_{u^{\prime}}$ the deformations are isomorphic and the isomorphism is unique since the automorphism groups are trivial on a dense open set. Therefore the glueing is unique and the compatibility relation is trivially satisfied. By [W], this family is universal at every point because $H^{0}\left(S_{u}, \Theta_{u}\right)=0$ for every $u$.

Now let $\left(0, \ldots, 0, \alpha_{i}, 0, \ldots, 0\right)$ be a fixed point. We may suppose that $\alpha_{i}=1$ and we choose a versal deformation over a polydisc $\Delta^{n}$. The group $L$ is generated by $\Lambda: \mathbb{P}^{n-1} \rightarrow \mathbb{P}^{n-1}$ defined by 


$$
\begin{aligned}
& \Lambda\left(\alpha_{0}, \ldots, \alpha_{i-1}, 1, \alpha_{i+1}, \ldots, \alpha_{n-1}\right) \\
& \quad=\left(\lambda^{-i} \alpha_{0}, \ldots, \lambda^{-1} \alpha_{i-1}, 1, \lambda \alpha_{i+1}, \ldots, \lambda^{n-1-i} \alpha_{n-1}\right) .
\end{aligned}
$$

By the universality the glueing is unique and completes the family over the singularities.

\section{References}

[B] F. A. Bogomolov, Surfaces of class VII-0 and affine geometry, Math. USSR-Izv. 21 (1983), 31-73.

[BR] M. Brunella, Feuilletages holomorphes sur les surfaces complexes compactes, prépublication 86 (1996), Université de Bourgogne, Dijon.

[DA] K. Dąbrowski, Kuranishi families for Hopf surfaces, Ann. Polon. Math. 45 (1985), 61-84.

[D1] G. Dloussky, Structure des surfaces de Kato, Mém. Soc. Math. France 112 (1984), no. 14.

[D2] - Sur la classification des germes d'applications holmorphes contractantes, Math. Ann. 280 (1988), 649-661.

[D3] -, Une construction élémentaire des surfaces d'Inoue-Hirzebruch, ibid. 280 (1988), 663-682.

[E1] I. Enoki, Surfaces of class VII $I_{0}$ with curves, Tôhoku Math. J. 33 (1981), 453492.

[E2] —, Deformations of surfaces containing global spherical shells, in: Classification of Algebraic and Analytic Manifolds (Katata, 1982), Progr. Math. 39, Birkhäuser, 1983, 45-64.

[H] J. H. Hubbard and R. W. Oberste-Vorth, Hénon mappings in the complex domain I: The global topology of dynamical space, Publ. Math. IHES 79 (1994), $5-46$.

[I] M. Inoue, New surfaces with no meromorphic functions II, in: Complex Analysis and Algebraic Geometry, W. L. Baily and T. Shioda (ed.), Cambridge Univ. Press and Iwanami Shoten Publ., 1977, 91-106.

[IKO] M. Inoue, S. Kobayashi and T. Ochiai, Holomorphic affine connections on compact complex surfaces, J. Fac. Sci. Univ. Tokyo Sci. IA 27 (1980), 247-264.

[KA] M. Kato, Compact complex manifolds containing "global spherical shells", in: Proc. Internat. Sympos. Algebraic Geometry (Kyoto, 1977), Kinokuniya Book Store, Tokyo, 1978, 45-84.

[KW] Y. Kawamata, On deformations of compactifiable complex manifolds, Math. Ann. 235 (1978), 247-265.

[KO] K. Kodaira, On the structure of compact complex analytic surface, I, Amer. J. Math. 86 (1964), 751-798; II, ibid. 88 (1966), 682-721.

[KH1] F. Kohler, Feuilletages holomorphes singuliers et déformations sur les surfaces contenant une coquille sphérique globale, thèse, Université d'Aix-Marseille I, 1994.

[KH2] - Feuilletages holomorphes singuliers sur les surfaces contenant une coquille sphérique globale, Ann. Inst. Fourier 45 (1995), 161-182; erratum, ibid., 46 (1996), 589.

[KH3] —, Feuilletages holomorphes singuliers sur les surfaces contenant une coquille sphérique globale, in: Estado actual y persectivas en singularidades de ecuaciones 
diferenciales y foliaciones holomorfas (Medina, 1995), J. Mozo (ed.), Serie Ciencias 15, Secretariado de Publicacioes e intercambio cientifico, 1997, 143-159.

[LYZ] J. Li, S. T. Yau and F. A. Zheng, A simple proof of Bogomolov's theorem on class $V I I_{0}$ surfaces with $b_{2}=0$, Illinois J. Math. 34 (1990), 217-220.

[N1] I. Nakamura, On surfaces of class VII $I_{0}$ with curves, Invent. Math. 78 (1984), 393-443.

[N2] -, On surfaces of class VII $I_{0}$ with curves II, Tôhoku Math. J. 42 (1990), 475-516.

[NA] M. Namba, Automorphism groups of Hopf surfaces, ibid. J. 26 (1974), 133-152.

[T] A.-D. Teleman, Projectively flat surfaces and Bogomolov's theorem on VII surfaces, Internat. J. Math. 5 (1994), 253-264.

[W] J. Wavrik, Obstructions to the existence of a space of moduli, in: Global Analysis, Princeton Univ. Press, Princeton, 1969, 403-413.

U.R.A. 225 C.N.R.S.

Centre de Mathématiques et d'Informatique

Université d'Aix-Marseille I

39, rue F. Joliot-Curie

F-13453 Marseille Cedex 13, France

E-mail: dloussky@gyptis.univ-mrs.fr
Département de Mathématiques

Université d'Angers

2, Bd Lavoisier

F-49045 Angers Cedex, France 